\title{
Complement activation on endothelium initiates antibody-mediated acute lung injury
}

\author{
Simon J. Cleary, ${ }^{1}$ Nicholas Kwaan, ${ }^{1}$ Jennifer J. Tian, ${ }^{1}$ Daniel R. Calabrese, ${ }^{1,2}$ Beñat Mallavia, ${ }^{1}$ Mélia Magnen, ${ }^{1}$ John R. Greenland, ${ }^{1,2}$ \\ Anatoly Urisman, ${ }^{3}$ Jonathan P. Singer, ${ }^{1}$ Steven R. Hays, ${ }^{1}$ Jasleen Kukreja, ${ }^{4}$ Ariel M. Hay, ${ }^{5}$ Heather L. Howie, ${ }^{5}$ Pearl Toy, ${ }^{6}$ \\ Clifford A. Lowell, ${ }^{6}$ Craig N. Morrell, ${ }^{7}$ James C. Zimring, ${ }^{5}$ and Mark R. Looney, ${ }^{1,6}$ \\ 1Department of Medicine, UCSF, San Francisco, California, USA. ${ }^{2}$ Veterans Affairs Healthcare System, San Francisco, California, USA. ํㅗㄹepartment of Pathology and ${ }^{4}$ Department of Surgery, UCSF, San \\ Francisco, California, USA. ${ }^{5}$ Department of Pathology, University of Virginia School of Medicine, Charlottesville, Virginia, USA. ${ }^{6}$ Department of Laboratory Medicine, UCSF, San Francisco, California, USA. \\ ${ }^{7}$ Aab Cardiovascular Research Institute, University of Rochester School of Medicine and Dentistry, Rochester, New York, USA.
}

\begin{abstract}
Antibodies targeting human leukocyte antigen (HLA)/major histocompatibility complex (MHC) proteins limit successful transplantation and transfusion, and their presence in blood products can cause lethal transfusion-related acute lung injury (TRALI). It is unclear which cell types are bound by these anti-leukocyte antibodies to initiate an immunologic cascade resulting in lung injury. We therefore conditionally removed MHC class I (MHC I) from likely cellular targets in antibodymediated lung injury. Only the removal of endothelial MHC I reduced lung injury and mortality, related mechanistically to absent endothelial complement fixation and lung platelet retention. Restoration of endothelial MHC I rendered MHC Ideficient mice susceptible to lung injury. Neutrophil responses, including neutrophil extracellular trap (NET) release, were intact in endothelial MHC I-deficient mice, whereas complement depletion reduced both lung injury and NETs. Human pulmonary endothelial cells showed high HLA class I expression, and posttransfusion complement activation was increased in clinical TRALI. These results indicate that the critical source of antigen for anti-leukocyte antibodies is in fact the endothelium, which reframes our understanding of TRALI as a rapid-onset vasculitis. Inhibition of complement activation may have multiple beneficial effects of reducing endothelial injury, platelet retention, and NET release in conditions where antibodies trigger these pathogenic responses.
\end{abstract}

\section{Introduction}

Human leukocyte antigens (HLAs), the major histocompatibility complex (MHC) proteins in humans, are frequently the targets of antibodies that are a major barrier to the success of blood transfusion and organ transplantation therapies. One complication known to arise from anti-HLA antibodies is a leading cause of mortality from blood transfusions - transfusion-related acute lung injury (TRALI), a noncardiogenic form of pulmonary edema developing within 6 hours of transfusion (1). Research into the mechanistic basis of TRALI has directly led to successful mitigation strategies including exclusion from the donor pool of high plasma volume products from previously pregnant donors who have a high incidence of anti-HLA antibody positivity (2); however, substantial risk remains (3). It also remains unclear how anti-HLA antibodies induce microvascular pathology and lung injury, and there is additional unmet need for improved pathological understanding to drive therapeutic innovation for anti-HLA-mediated rejection of solid organ transplants (4).

A major unresolved issue in TRALI pathogenesis research has been determining the site of antibody binding required for initia-

Conflict of interest: The authors have declared that no conflict of interest exists. Copyright: @ 2020, American Society for Clinical Investigation.

Submitted: March 16, 2020; Accepted: July 22, 2020; Published: October 5, 2020.

Reference information: J Clin Invest. 2020;130(11):5909-5923.

https://doi.org/10.1172/JCl138136. tion of lung injury. TRALI is classically associated with anti-granulocyte antibodies (5), and the binding of antibodies to monocytes has also been suggested to be critical for induction of anti-HLA class II-mediated TRALI (6). However, nonleukocytic cells also express HLAs. In fact, a case report describing graft-localized TRALI in a unilateral lung transplant recipient transfused with blood containing lung donor-specific anti-HLA class I antibodies (7), and an irradiation and bone marrow reconstitution study in mice (8), point toward antibody binding to a nonhematopoietic cell as a probable initiating event in TRALI.

In this study, we aimed to identify the critical binding site of the antibody initiating lung injury in a mouse model of antiMHC class I-mediated (anti-MHC I-mediated) TRALI, and then to determine the immediate downstream immunologic events leading to lung barrier disruption. To accomplish this aim, we first used a genetic approach for lineage-restricted deletion of $B 2 m$ (9). The $B 2 m$ gene encodes $\beta$-2-microglobulin ( $B 2 m)$, which is required for surface expression of MHC I proteins $(10,11)$. In this manner, MHC I proteins were selectively removed from the surface of each of the cell types likely to be bound by antibody following intravenous infusion: endothelial cells, different myeloid leukocyte subsets, or the megakaryocyte-platelet lineage. To support findings in mice with conditional MHC I removal, we also developed a new mouse strain enabling lineage-restricted restoration of B2m expression in order to study the effect of restoring MHC I exclusively on one cell type. Combining insights from genetic and 
pharmacological studies with intravital lung microscopy, we were able to identify the site of antibody binding critical for lung injury. We also found distinct pathways leading to lung platelet retention and neutrophil extracellular trap (NET) formation, and an intervention that prevents immunologic injury to the lung. These findings have direct relevance to blood transfusion therapy, as well as other conditions where host cells become the targets of antibodies resulting in inflammation and organ injury.

\section{Results}

C57BL/6-congenic mice with $\mathrm{H}-2^{d}$ MHC I enable anti-MHC Imediated lung injury studies on an otherwise C57BL/6 background. Experimental TRALI involves i.v. injection of the 34-1-2S monoclonal antibody (anti-H-2d, known to bind $\mathrm{K}^{\mathrm{d}}$ and $\mathrm{D}^{\mathrm{d}}$ ) into mice possessing $\mathrm{H}-2^{\mathrm{d}}$ haplotypes, such as BALB/c mice (12). Curiously, anti-H- $2^{\mathrm{d}}$ can bind to $\mathrm{K}^{\mathrm{b}}$ expressed by $\mathrm{C} 57 \mathrm{BL} / 6$ (B6) mice (ref. 13 and Supplemental Figure 1, A-F; supplemental material available online with this article; https://doi.org/10.1172/JCI138136DS1). However, B6 mice are resistant to lung injury (8), unless the mice are $\mathrm{T}$ cell depleted and given high doses of anti- $\mathrm{H}-2^{\mathrm{d}}$ in combination with an additional anti-H-2 $\mathrm{K}^{\mathrm{b}}$ antibody (14). B6.C- $\mathrm{H} 2^{d} / b B y J$ $\left(\mathrm{B} 6 . H 2^{d}\right)$ mice are on a B6 background except for their BALB/cderived $H 2^{d}$ locus, which encodes the $\mathrm{H}-2^{\mathrm{d}}$ type MHC I proteins, allowing the transfer of the $\mathrm{H}-2^{\mathrm{d}}$ type to $\mathrm{B} 6$ background strains after 2 crosses rather than the more than 10 backcrosses required to produce congenic mice from hybrid crosses. We hypothesized that $\mathrm{B} 6 . \mathrm{H} 2^{d}$ mice would develop anti-H-2 ${ }^{\mathrm{d}}$-mediated lung injury using the same protocol (LPS priming followed by antibody injection) as that used previously with the BALB/c strain (15). Similarly to $\mathrm{BALB} / \mathrm{c}$ mice, $\mathrm{B} 6 . \mathrm{H}^{d}{ }^{d}$ mice treated with i.v. anti-H-2 ${ }^{\mathrm{d}}$ antibody developed lung vascular permeability and pulmonary edema (Figure $1, \mathrm{~A}$ and $\mathrm{B}$ ). This acute lung injury mostly resolved 24 hours after anti-H-2d injection (Supplemental Figure 2, A and B). When a higher dose of anti-H-2 ${ }^{\mathrm{d}}$ was given, $\mathrm{B} 6 . \mathrm{H} 2^{d}$ mice showed greater susceptibility to mortality than BALB/c mice (Figure 1C).

Identification of the cell type bound by antibody to initiate lung injury. $\mathrm{B} 2 \mathrm{~m}$ is critical for cell surface expression of the MHC I proteins, and a $\mathrm{B} 2 \mathrm{~m}^{\text {flox }}$ strain has recently been developed that allows the lineage-restricted ablation of MHC I from cell surfaces when further crossed to existing promoter-specific Cre strains (9). $\mathrm{B} 2 \mathrm{~m}^{\mathrm{f} / \mathrm{fl}}$ mice were crossed to strains expressing VE-cadherin-Cre (VE-Cad-Cre) to target endothelial cells (16), PF4-Cre to target the megakaryocyte-platelet lineage (17), LysM-Cre to target neutrophils and monocytes (18), and CX3CR1-Cre to target Ly6C lo monocytes $(19,20)$. Additionally, all $\mathrm{B} 2 \mathrm{~m}^{\mathrm{fl} / \mathrm{fl}}$ mice were crossed to homozygosity with $\mathrm{B} 6 . \mathrm{H} 2^{d}$ mice for full $\mathrm{H}-2^{\mathrm{d}} \mathrm{MHC}$ I expression (Supplemental Figure 3, A-D).

Targeted removal of $\mathrm{B} 2 \mathrm{~m}$ from endothelial cells (VE-Cad-Cre $\times$ $\left.\mathrm{B} 2 \mathrm{~m}^{\mathrm{f} / \mathrm{II}}\right)$ resulted in efficient reductions in surface $\mathrm{MHC}$ I expression on pulmonary endothelial cells relative to Cre-negative littermates (Figure 1, D and E). LPS-primed endothelial MHC I-deficient mice given anti-H-2 ${ }^{\text {d }}$ i.v. were protected from lung vascular permeability and edema (Figure 1, F and G), and at a high anti- $\mathrm{H}-2^{\mathrm{d}}$ dose, were protected from mortality (Figure $1 \mathrm{H}$ ), and alveolar flooding assessed using lung histology (Supplemental Figure 4, A and B). These findings point toward anti-H- ${ }^{\mathrm{d}}$ binding to endothelial $\mathrm{MHC}$ I being a critical event required to initiate full TRALI pathology.
Ablation of $\mathrm{B} 2 \mathrm{~m}$ in the megakaryocyte-platelet lineage $\left(\mathrm{PF} 4-\mathrm{Cre} \times \mathrm{B} 2 \mathrm{~m}^{\mathrm{f} / \mathrm{f}}\right.$ ) efficiently removed MHC I expression from the surface of blood platelets (Supplemental Figure 5, A and B), but PF4-Cre $\times B 2 \mathrm{~m}^{\mathrm{f} / \mathrm{fl}}$ mice showed no reduction in anti-H-2 ${ }^{\mathrm{d}}-$ induced lung injury relative to Cre-negative controls (Supplemental Figure 5, C and D).

Using LysM-Cre $\times \mathrm{B} 2 \mathrm{~m}^{\mathrm{f} / \mathrm{fl}}$ and CX3CR1-Cre $\times \mathrm{B} 2 \mathrm{~m}^{\mathrm{f} / \mathrm{fl}}$ mice, we measured the extent of removal of surface MHC I from neutrophils, as well as Ly6C ${ }^{\text {lo }}$, Ly6C ${ }^{\text {mid }}$, and Ly6 $\mathrm{C}^{\text {hi }}$ monocytes (Supplemental Figure $6 \mathrm{~A}$ ). LysM-Cre $\times \mathrm{B} 2 \mathrm{~m}^{\mathrm{f} / \mathrm{fl}}$ mice had greater than $70 \%$ reductions in surface $\mathrm{MHC}$ I on neutrophils, and also reductions on $\mathrm{Ly}^{6} \mathrm{C}^{\mathrm{hi}}$ and, to a lesser extent, Ly6 $\mathrm{C}^{\mathrm{lo}}$ monocytes ( $>60 \%$ and $>35 \%$ reductions, Supplemental Figure 6 , B and C), but when challenged with anti-H-2 $2^{\mathrm{d}}$ antibody, LysM-Cre $\times \mathrm{B} 2 \mathrm{~m}^{\mathrm{fl} / \mathrm{fl}}$ mice had unaltered lung injury compared with Cre-negative littermates (Supplemental Figure 6, D and E).

Relative to Cre-negative controls, CX3CR1-Cre $\times \mathrm{B} 2 \mathrm{~m}^{\mathrm{fl} / \mathrm{fl}}$ mice had efficient reductions in the notably high surface expression of MHC I on Ly6 $\mathrm{C}^{\mathrm{lo}}$ monocytes and had unaltered MHC I expression on other myeloid cell subsets (Supplemental Figure 6, F and G). These observations are despite positive CX3CR1 expression on Ly $6 \mathrm{C}^{\text {hi }}$ monocytes - likely a result of CX3CR1 expression too late in the development of these short-lived cells for removal of existing MHC I proteins (20). When challenged with anti- $\mathrm{H}-2^{\mathrm{d}}$ antibody, CX3CR1-Cre $\times \mathrm{B} 2 \mathrm{~m}^{\mathrm{f} / \mathrm{fl}}$ mice had no reduction in the extent of lung injury relative to Cre-negative littermate controls, but in fact had elevated pulmonary edema relative to controls (Supplemental Figure 6, $\mathrm{H}$ and I).

Together, these data suggest that antibody engagement of platelet, neutrophil, or Ly6 $\mathrm{C}^{\text {lo }}$ monocyte MHC I are dispensable for the initiation of lung injury, and that the presence of highly expressed Ly $6 \mathrm{C}^{\text {lo }}$ monocyte MHC I may be protective against antibody-mediated lung injury.

Endothelial cell-restricted restoration of MHC I expression restores susceptibility to lung injury. For further testing of the importance of endothelial cell versus leukocyte MHC I in antibody-mediated lung injury, a new mouse strain was created to allow the conditional reversion of globally inverted $B 2 m$ ( $\mathrm{B} 2 \mathrm{~m}^{\mathrm{inv} / \mathrm{inv}}$ mouse). In the endogenous $B 2 m$ locus of this mouse, exons 2 and 3 were inverted and loxP-flanked for conditional reversion and therefore restoration of MHC I expression after Cre recombination (Figure 2A). These mice were also crossed onto the B6.H2 ${ }^{d}$ background for susceptibility to anti-H-2 ${ }^{\mathrm{d}}-$ mediated lung injury. Mice homozygous for the modified allele (B2m ${ }^{\text {inv }}$ ) are globally deficient in B2m and therefore MHC I, but in VE-Cad-Cre $\times \mathrm{B} 2 \mathrm{~m}^{\text {inv/inv }}$ mice, recombination inverts $B 2 m$ to its natural orientation to restore full MHC I expression on endothelial cells (Figure 2, B and C), but not on Cre-negative cells such as leukocytes (Figure 2, D and E).

In contrast to injury-resistant $\mathrm{B} 2 \mathrm{~m}^{\mathrm{inv} / \mathrm{inv}}$ mice, VE-Cad-Cre $\times \mathrm{B} 2 \mathrm{~m}^{\text {inv/inv }}$ littermates challenged with i.v. injection of anti-H-2 developed robust lung vascular permeability and edema responses (Figure 2, F and G), and died when challenged with a higher anti-H-2 ${ }^{\mathrm{d}}$ dose (Figure $2 \mathrm{H}$ ). This result demonstrates that the expression of antigen exclusively on the endothelium is sufficient to confer susceptibility to anti-MHC I-mediated lung injury, and that expression of MHC I on other cells including leukocytes is not a requirement for lung injury responses in this model. 
LPS i.p. $+1 \mathrm{mg} / \mathrm{kg}$ anti-H-2d/isotype i.v.

$\mathbf{A}$

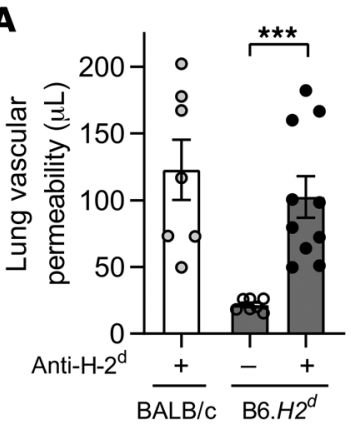

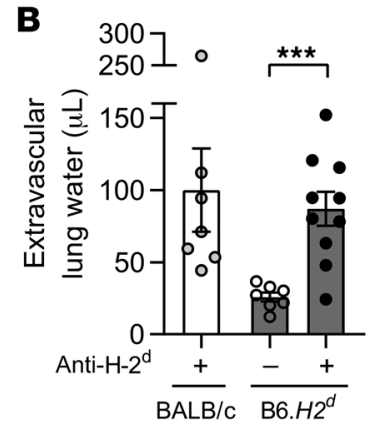

B6. $H 2^{d} \times B 2 m^{f / / f l}$
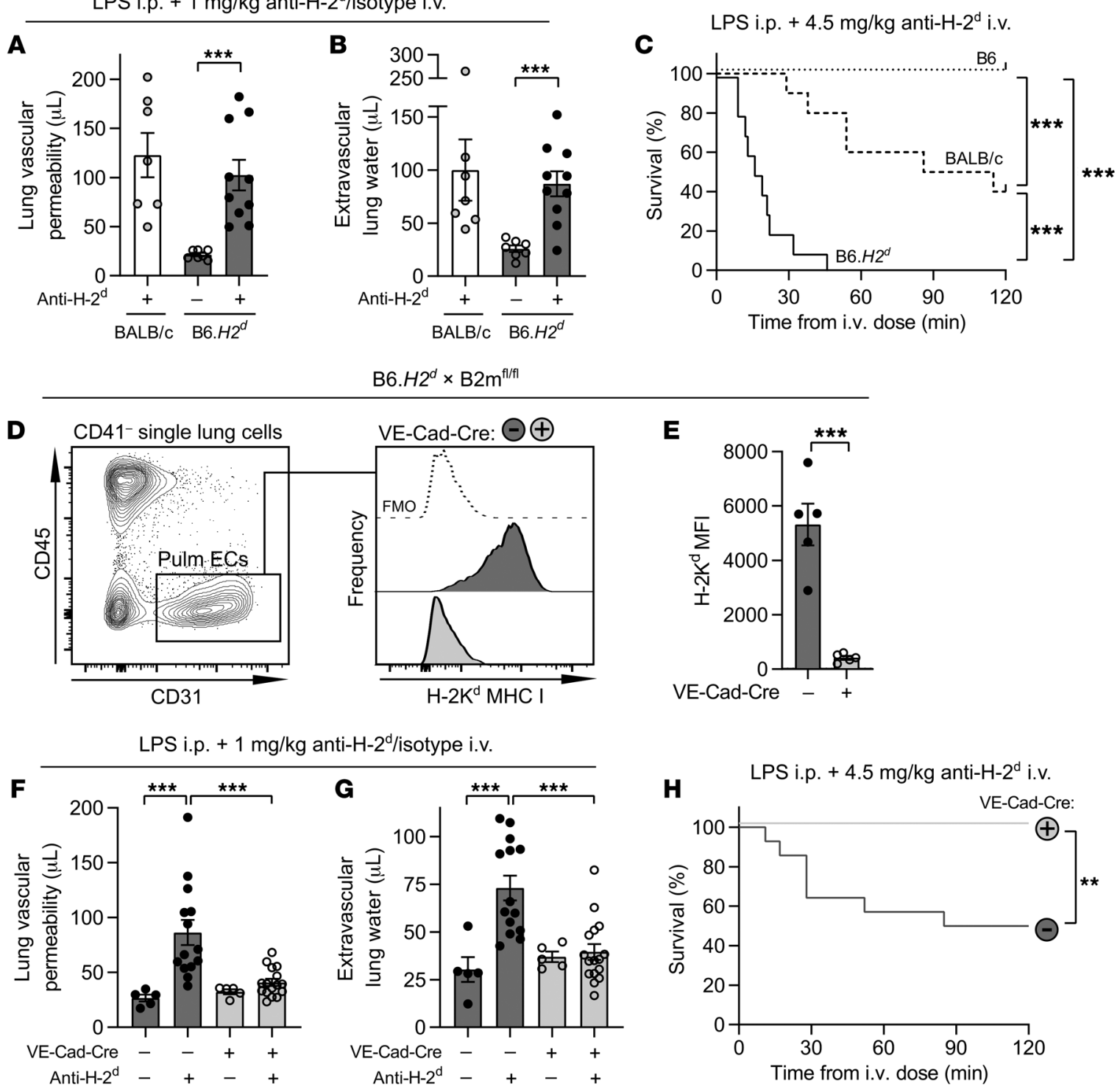

Figure 1. Removal of MHC I from endothelium is protective in anti-MHC I-mediated lung injury. LPS-primed BALB/C or B6. H2 $2^{d}$ mice were administered isotype or anti-H-2 ${ }^{d}$ antibody i.v. for measurements of (A) lung vascular permeability and (B) extravascular lung water 2 hours after antibody injection

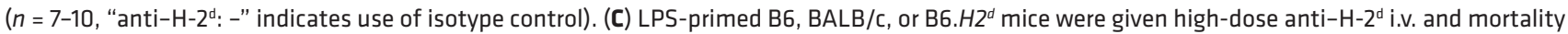
was assessed over 2 hours after injection $(n=10)$. Lungs of $B 6 . H 2^{d} \times B 2 \mathrm{~m}^{\mathrm{fl} / \mathrm{fl}}$ mice either positive or negative for VE-Cad-Cre were dissociated and used for measurement of $\mathrm{H}-2 \mathrm{~K}^{\mathrm{d}} \mathrm{MHC}$ class I (MHC I) expression on pulmonary endothelial cells. (D) Gating strategy and representative histograms and (E) median fluorescence intensity $(\mathrm{MFI})$ quantification $(n=5)$. FMO, fluorescence minus one. Separate mice were given LPS i.p. and isotype or anti-H-2 ${ }^{\mathrm{d}} \mathrm{i} . \mathrm{V}$. and used for assessment of (F) lung vascular permeability and (G) extravascular lung water at 2 hours after i.v. anti-H-2 ${ }^{\mathrm{d}}$ antibody (isotype controls, $n=5$; + antiH- $2^{d}$ i.v.: $\mathrm{Cre}^{-} n=14, \mathrm{Cre}^{+} n=16$ ). (H) Additional mice were given LPS and high-dose anti-H-2 ${ }^{\mathrm{d}}$ antibody i.v. and monitored for survival ( $\left.n=14\right)$. Mean \pm SEM. ${ }^{* *} P<0.01$; ${ }^{* *} P<0.001$ by 2-way ANOVA with Holm's test (A, B, F, and $\mathbf{G}$ ), log-rank test (C and $\mathbf{H}$ ) with Bonferroni's correction (C), or unpaired $t$ test (E).

High surface expression of MHC I on pulmonary endothelial cells. Having observed high MHC I levels on pulmonary endothelial cells and blood monocytes, we directly compared surface MHC I expression across mouse lung cells. Both monocytes and endothelial cells expressed the highest levels of surface MHC/HLA class I in mouse lung cells, and LPS priming upregulated only lung neutrophil and monocyte surface MHC I expression in mice (Supplemental Figure $7, \mathrm{~A}^{-} \mathrm{C}$ ). These results, and the location of the lung microvasculature as the first capillary bed encountered by an i.v. injection, indicate that the pulmonary endothelium is a likely binding site for i.v.-infused anti-MHC I antibodies.
Lung intravital imaging of early immune events leading to lung injury. To assess the kinetics and distribution of antibody retention in the lungs, we directly imaged the deposition of PE-conjugated anti- $\mathrm{H}-2^{\mathrm{d}}$ in the lungs of lung injury-susceptible B6. $H 2^{d}$ mice and lung injury-resistant $\mathrm{B} 6$ controls. B6. $H 2^{d}$ mice showed greater retention of anti-H-2 ${ }^{\mathrm{d}}$ in the pulmonary vasculature (Figure 3, A and B, and Supplemental Video 1), consistent with a requirement for the more avid antibody binding to $\mathrm{H}-2^{\mathrm{d}}$ versus $\mathrm{H}-2^{\mathrm{b}}$ type $\mathrm{MHC}$ I proteins for lung antibody retention.

Myeloid leukocytes and platelets are implicated as cellular effectors of TRALI $(12,15)$, so we simultaneously imaged these 

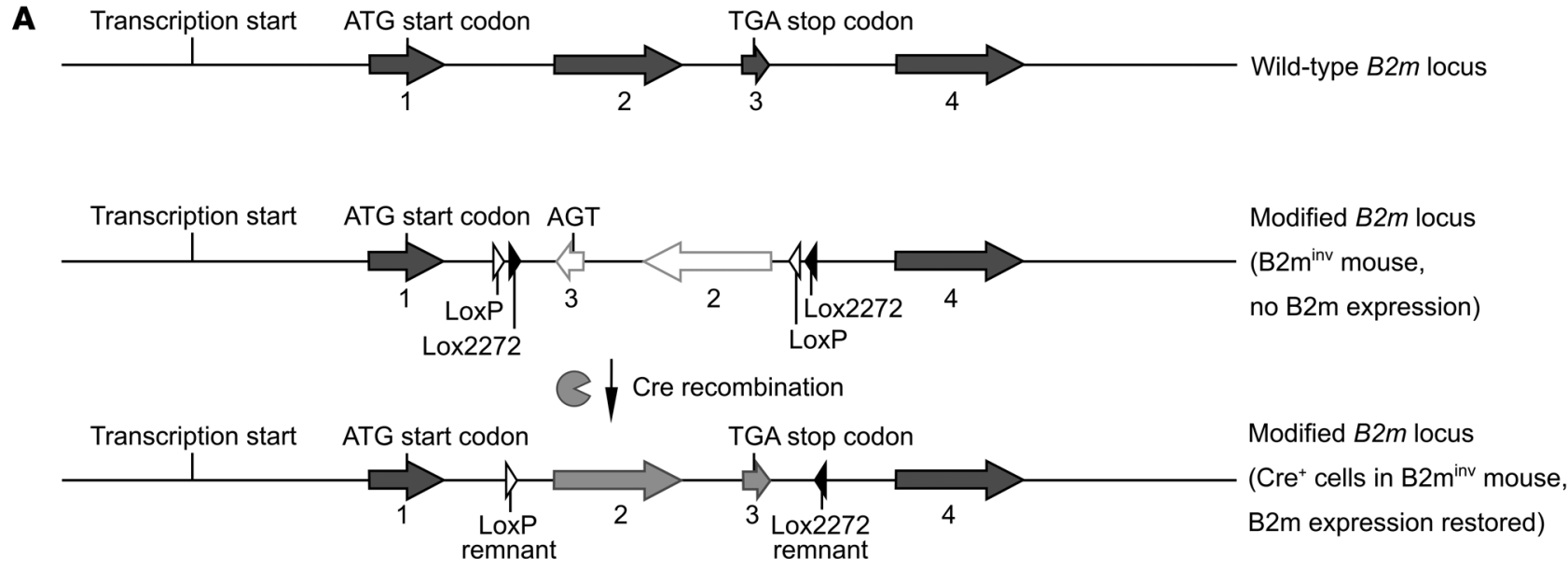

B6. $H 2^{d}$

B6. $H 2^{d} \times B 2 m^{\text {invlinv }}$

B $6 . H 2^{d} \times$ VE-Cad-Cre $\times B 2 m^{\text {inv/inv }}$

Pulmonary endothelial cells

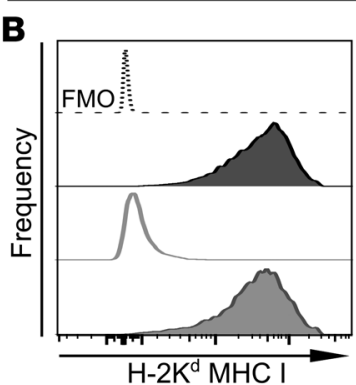

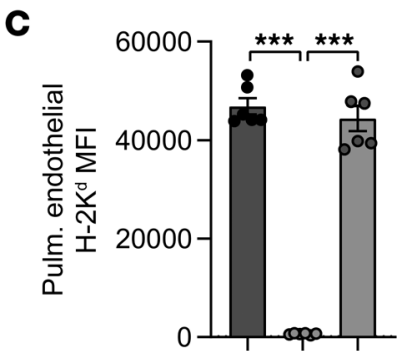

B2m + +t inv/inv

VE-Cad-Cre - _ +
LPS i.p. $+1 \mathrm{mg} / \mathrm{kg}$ anti-H-2 ${ }^{\text {d }}$ i.v.
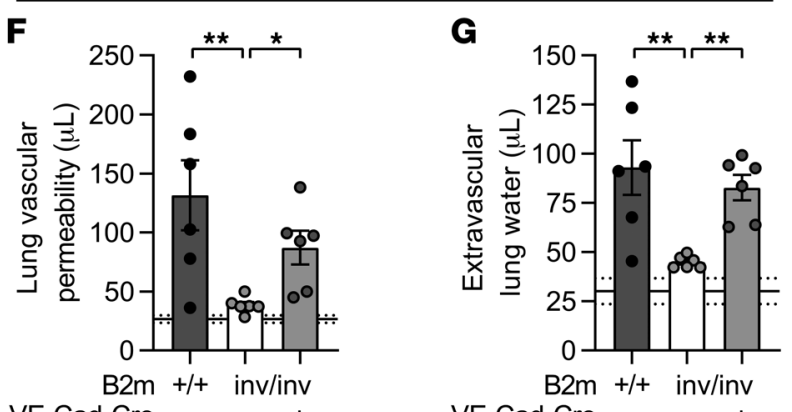

VE-Cad-Cre - _ +
Blood leukocytes
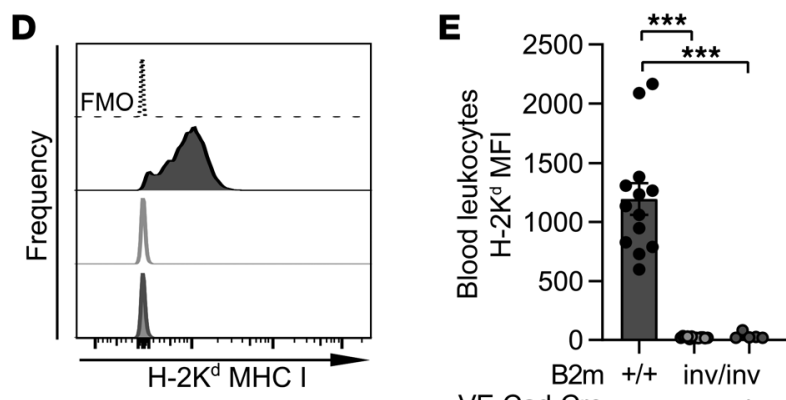

VE-Cad-Cre - - + +

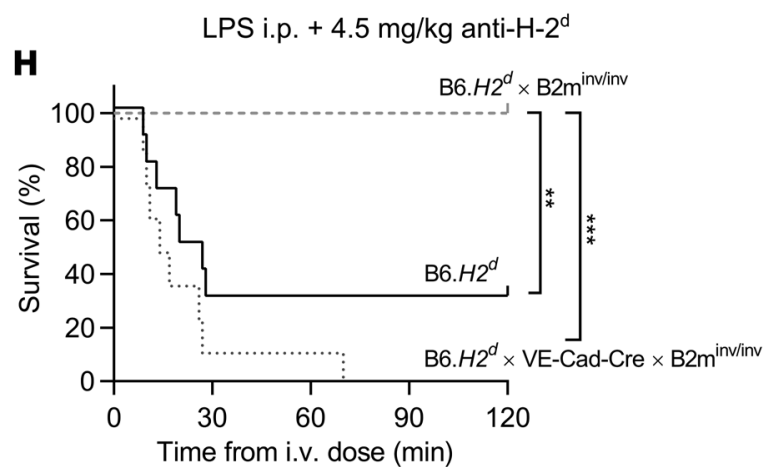

Figure 2. Restoration of MHC I exclusively on endothelium is sufficient to induce susceptibility to anti-MHC I-mediated lung injury. B2minv/inv mice were created in which exons 2 and 3 of the B2m locus were inverted and flanked by loxP and lox 2272 sites to permit conditional restoration of B2m expression and therefore surface MHC I expression in response to Cre recombination. (A) Schematic showing WT and modified B2m loci with and without Cre recombination. Lungs from B6. $2^{d}$ reference controls, B6. $H 2^{d}$-background B2 ${ }^{\text {inv/inv }}$ Cre-negative mice, and their VE-Cad-Cre $\times$ B2m ${ }^{\text {inv/inv }}$ littermates were dissociated for measurement of $\mathrm{H}-2 \mathrm{~K}^{\mathrm{d}} \mathrm{MHC}$ I expression on $\mathrm{CD} 41^{-}, \mathrm{CD} 45^{-}, \mathrm{CD} 31^{+}$pulmonary endothelial cell surfaces. (B) Representative histograms showing pulmonary endothelial $\mathrm{H}-2 \mathrm{~K}^{\mathrm{d}}$ expression, and (C) quantification of median fluorescence intensity (MFI) of pulmonary endothelial surface $\mathrm{H}-2 \mathrm{~K}^{\mathrm{d}}(n=6)$. Blood was also tested to confirm $\mathrm{H}-2 \mathrm{~K}^{\mathrm{d}} \mathrm{MHC}$ I removal from $\mathrm{CD} 45^{+}$leukocytes in B2m ${ }^{\text {inv/inv }}$ mice. (D) Representative leukocyte $\mathrm{H}-2 \mathrm{~K}^{d}$ histograms, and (E) quantification of leukocyte $\mathrm{H}-2 \mathrm{~K}^{d} \mathrm{MFI}\left(\mathrm{B} 6 . \mathrm{H} 2^{d}\right.$ and VE-Cad-Cre,$\left.n=13 ; \mathrm{VE}-\mathrm{Cad}_{-}-\mathrm{Cre}^{+}, n=5\right)$. Separate mice were primed with LPS and challenged with $1 \mathrm{mg} / \mathrm{kg}$ anti-H-2 ${ }^{\mathrm{d}}$ i.v. for measurements of (F) lung vascular permeability and (G) extravascular lung water (both $n=6$ ), or (H) $4.5 \mathrm{mg} / \mathrm{kg}$ anti-H-2 ${ }^{\mathrm{d}}$ i.v. for survival assessment (B6. $H 2^{d}$ and VE-Cad-Cre ${ }^{-}, n=10$; VE-Cad-Cre+, $n=8$ ). Mean \pm SEM. Horizontal lines in $\mathbf{F}$ and $\mathbf{G}$ are reference data from Figure 1 from mice given isotype control i.v. ${ }^{*} P<0.05 ;{ }^{* *} P<0.01 ;{ }^{* *} P<0.001$ by 1 -way ANOVA with Tukey's test (C, E, $\mathbf{F}$, and $\mathbf{G}$ ) or log-rank test with Bonferroni's correction (H). 
A

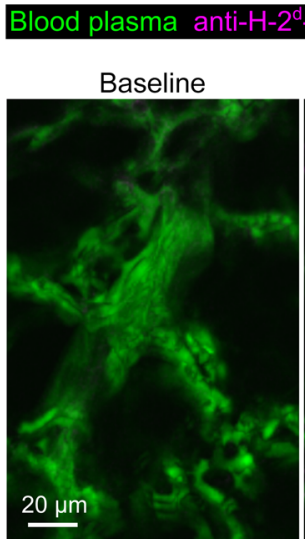

C

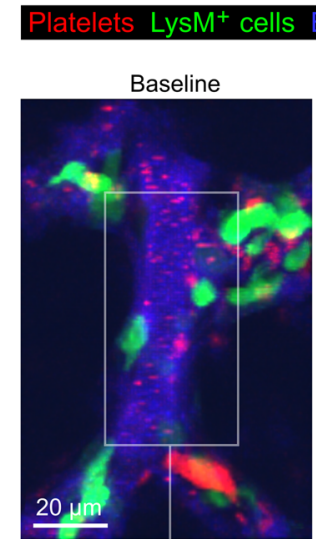

$\mathbf{F}$

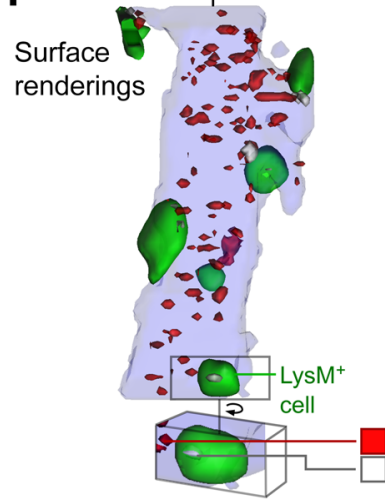

After anti-H-2d-PE i.v. into B6. $H 2^{d}$
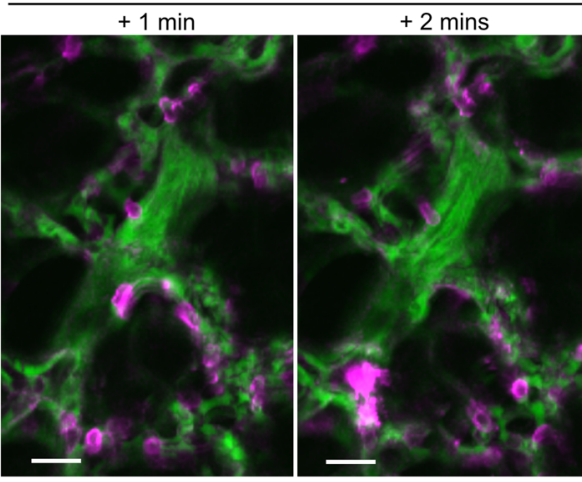

After anti-H-2 ${ }^{\text {di.v. }}$
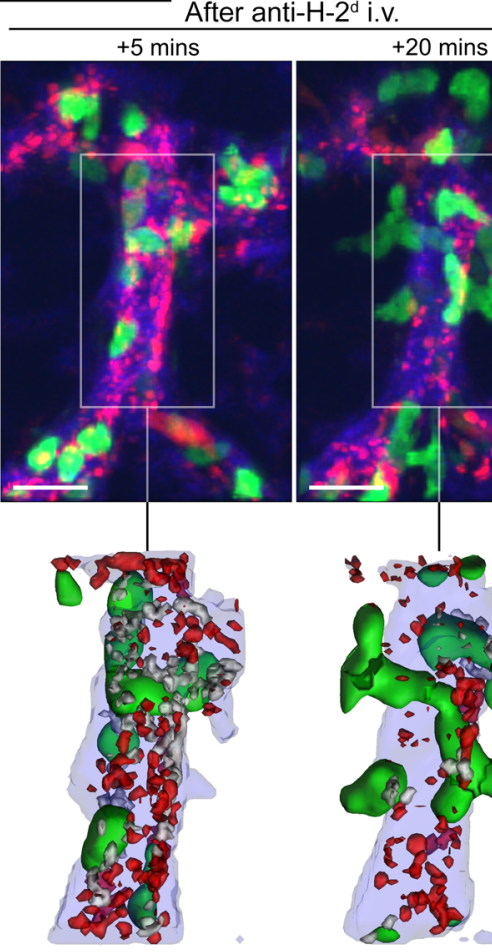

Platelet, not $\mathrm{LysM}^{+}$cell associated Platelet, $\mathrm{LysM}^{+}$cell associated
B

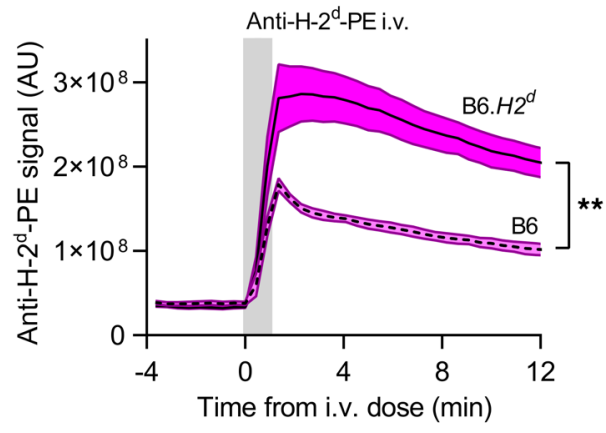

D

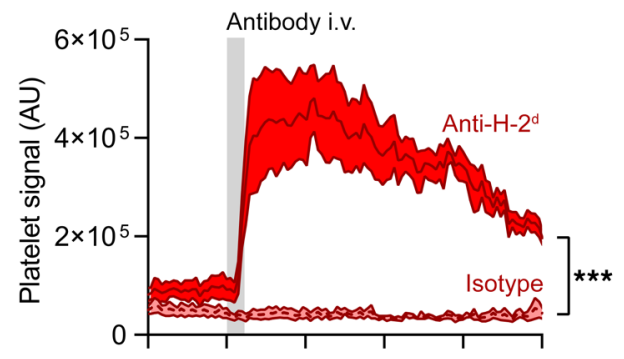

E

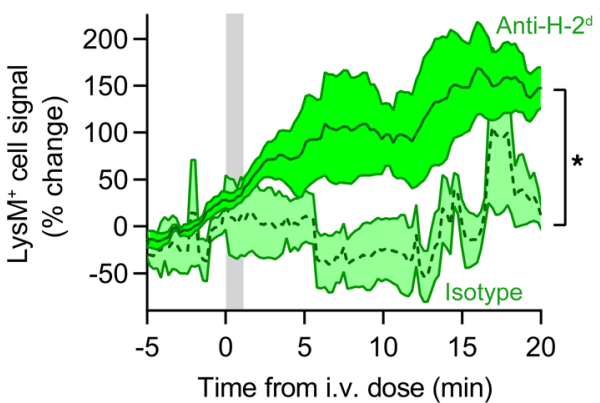

G

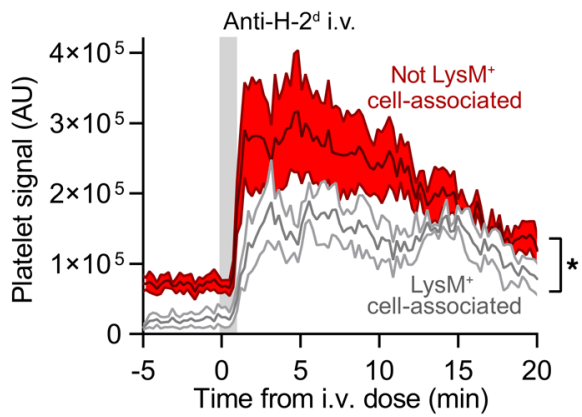

Figure 3. Spatially distinct retention of antibody, neutrophils, and platelets in the pulmonary vasculature during initiation of anti-MHC I-mediated lung injury. C57BL/6 (B6) or B6. H2d mice were given LPS i.p., $70 \mathrm{kDa}$ FITC-dextran i.v. (green), and then i.v. injections of anti-H-2d-PE (magenta) during 2-photon lung intravital imaging. (A) Representative images of antibody deposition responses in B6. $H 2^{d}$ pulmonary blood vessels and (B) quantification of anti-H-2 ${ }^{d}-\mathrm{PE}$ signal in imaged regions of lungs over time (B6, $n=3$; B6. $\left.H 2^{d}, n=4\right)$. LysM-GFP $\times$ PF4-Cre $\times$ Ai14 mice (LysM+ cells, green; PF4+ platelets, red) were given LPS i.p., Evans blue i.v. (blue), and then i.v. injections of anti- $\mathrm{H}-2^{\mathrm{d}}$ or isotype control during 2-photon lung intravital imaging $(n=4)$. (C) Responses to anti-H-2 $2^{\mathrm{d}}$ in a pulmonary blood vessel and quantification of (D) platelet and (E) Lys $\mathrm{M}^{+}$cell signal over time. (F) Surface renderings showing platelets associated with LysM+ ${ }^{+}$cell surfaces (white) and those not (red). (G) Quantification of platelet signal from Lys $\mathrm{M}^{+}$cell-associated platelets and those not associated with LysM+ cells. Mean \pm SEM. ${ }^{*} P<0.05 ;{ }^{* *} P<0.01 ;{ }^{* *} P<0.001$ by 2-way ANOVA with $P$ value for postbaseline effect of genotype (B), treatment ( $\mathbf{D}$ and $\mathbf{E})$, or Lys $\mathrm{M}^{+}$cell association (G).

cells in lungs using dual reporter mice (BALB/c-congenic LysMGFP $\times$ PF 4-Cre $\times$ Ai14; ref. 21). Platelet retention in lungs occurred within seconds of antibody injection, with platelets displaying margination toward the endothelium (Figure 3, C-E, and Supplemental Video 2). LysM $^{+}$cell retention was also detected over 20 minutes following anti-H-2d injection (Figure 3, C and E, and Sup- plemental Video 2). Rendering platelet and $\mathrm{LysM}^{+}$cell surfaces enabled quantification of the proportion of platelet signal arising from $\mathrm{LysM}^{+}$cell-associated platelets versus those not visibly associated with $\mathrm{LysM}^{+}$cells. The majority of platelet signal was found in locations distinct from $\mathrm{LysM}^{+}$cell surfaces, suggesting that platelet-leukocyte interactions are likely not solely responsible for 


\section{A}

B6. $H 2^{d} \times B 2 m^{f / f l l}$ mice

LPS i.p., $+1 \mathrm{mg} / \mathrm{kg}$ anti-H-2 $/$ isotype, +5 mins
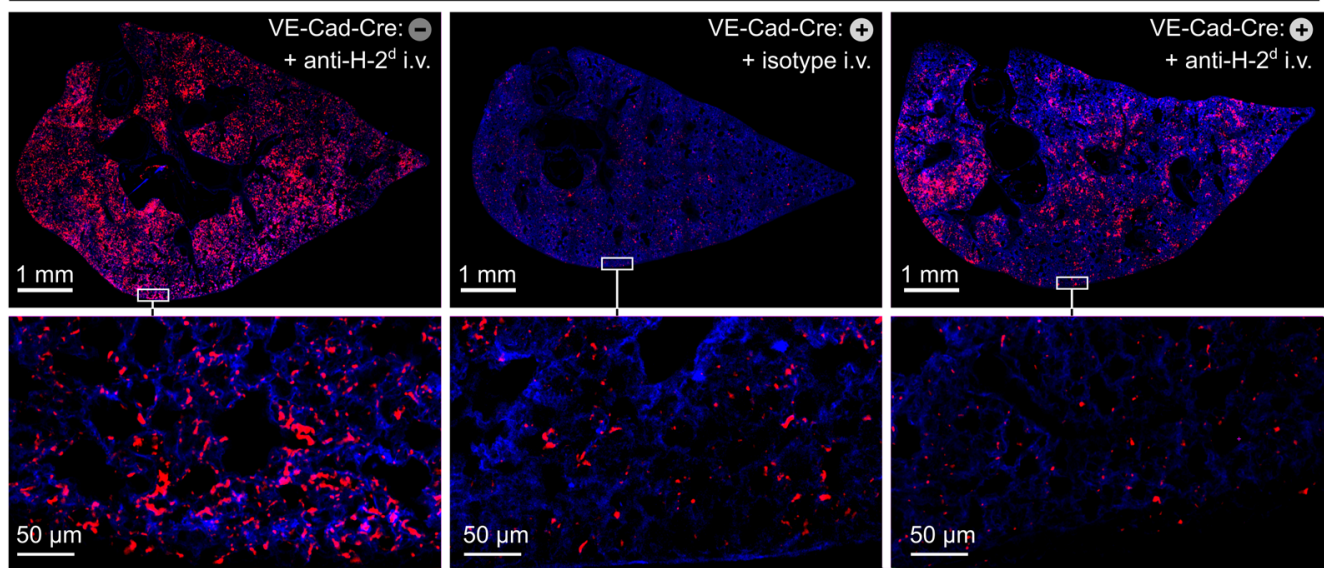

B

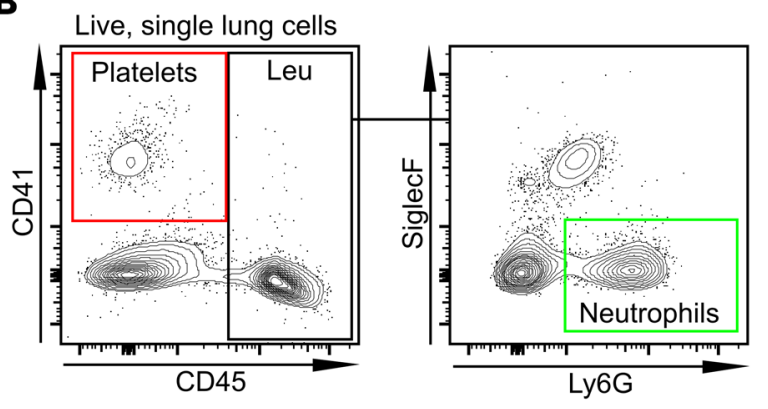

E

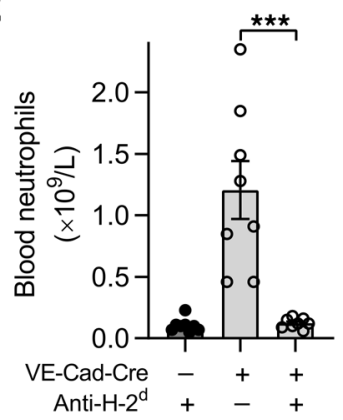

C

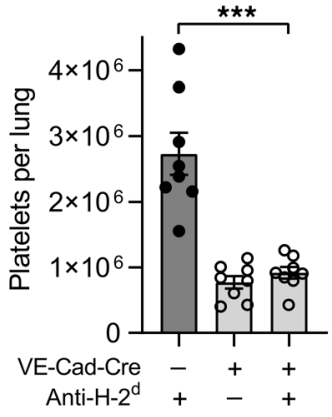

G

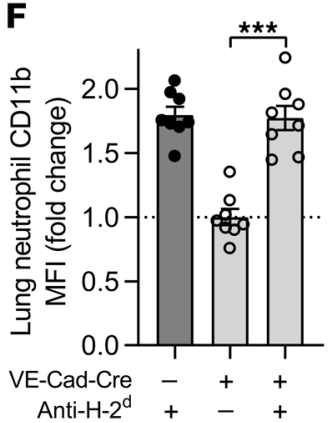

D
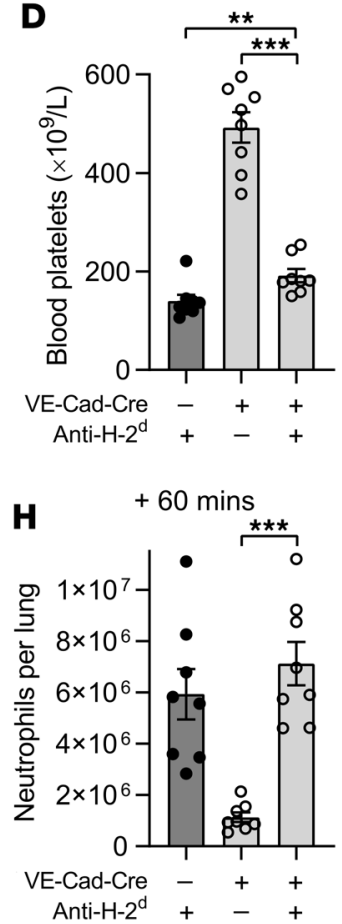

Figure 4. Removal of endothelial MHC I reduces lung platelet retention but not neutrophil activation in anti-MHC I-mediated lung injury. LPS-primed B6. $\mathrm{H}^{d} \times \mathrm{B} 2 \mathrm{~m}^{\mathrm{fl} / \mathrm{fl}}$ mice either positive or negative for VE-Cad-Cre were administered i.v. anti-H-2 ${ }^{\mathrm{d}}$ or isotype control. After 5 minutes, (A) lungs were collected for immunofluorescence staining for platelet CD41 (red) and collagen IV (blue) (representative of samples from 2 mice per group). In separate mice, blood plasma was sampled and lungs were collected for dissociation and flow cytometric analysis. (B) Gating strategy for identification of lung platelets and neutrophils, (C) quantification of platelets from lungs, and (D) blood. (E) Blood neutrophil counts and (F) lung neutrophil CD11b MFI normalized to isotype control group $P$ values. (C) Plasma neutrophil elastase (NE)-DNA complexes (NETs) quantified by ELISA. (H) Lung neutrophil counts using flow cytometry at 60 minutes after antibody injections. Mean $\pm \mathrm{SEM}$. ${ }^{*} P<0.05 ;{ }^{* *} P<0.01 ;{ }^{* *} P<0.001$ by 1 -way ANOVA and Dunnett's test vs. VE-Cad-Cre ${ }^{+}$ anti-H-2 $2^{\mathrm{d}}$-treated group $(\mathbf{C}-\mathbf{H}), n=8$.

lung platelet retention (Figure 3, F and G, and Supplemental Video 2). Platelet and neutrophil retention responses with similar kinetics could also be measured using flow cytometric analysis of dissociated whole lungs (Supplemental Figure 8, A-E). In addition to cell and platelet retention, we also live imaged transient ischemia, platelet aggregation, plasma extravasation, and the formation of neutrophil swarms in anti-MHC I-injured lungs (Supplemental
Figure 9, A and B, Supplemental Figure 10, and Supplemental Videos 3 and 4), all indicative of the rapid and severe immunologic events in the TRALI model. These experiments demonstrate that antibody-induced platelet and neutrophil retention in lungs are rapid, but spatially distinct processes.

Altered lung pathology in the absence of endothelial MHC I. Inspired by the lung imaging results, we next studied the effect of 
A B6. $H 2^{d} \times$ B2 $\mathrm{m}^{\mathrm{f} / \mathrm{f} / \mathrm{mice}}$, LPS i.p., $+1 \mathrm{mg} / \mathrm{kg}$ anti-H-2//isotype, +5 mins
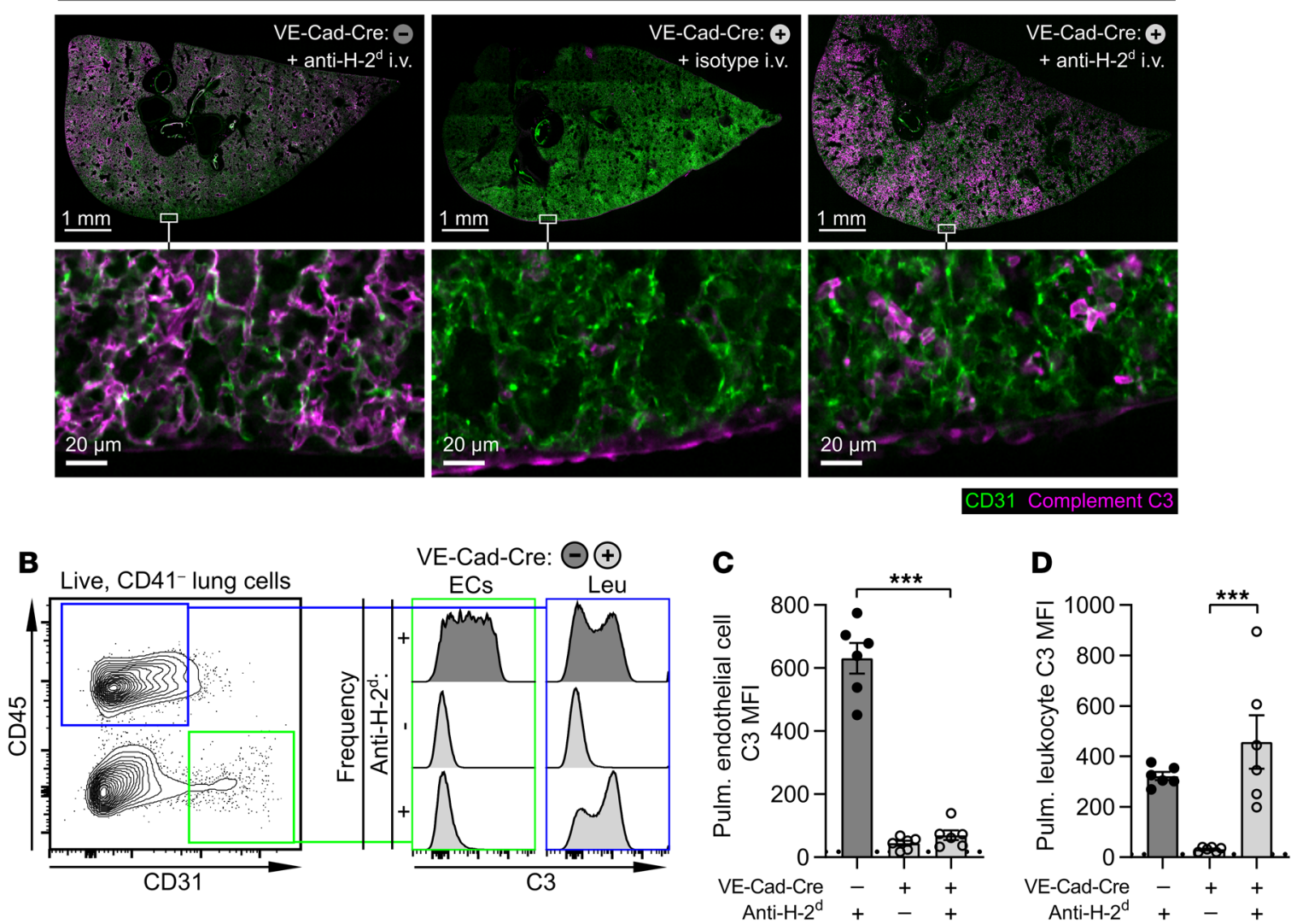

Figure 5. Removal of endothelial cell MHC I selectively spares pulmonary endothelial cells from fixation with complement in anti-MHC I-mediated lung injury. LPS-primed B6. $\mathrm{H}^{d} \times \mathrm{B} 2 \mathrm{~m}^{\mathrm{fl} / \mathrm{fl}}$ mice either positive or negative for $\mathrm{VE}-\mathrm{Cad}-\mathrm{Cre}$ were administered anti-H-2d or isotype control antibody i.v. and lungs collected 5 minutes later for (A) immunofluorescence staining of endothelial CD31 (green) and complement C3 (magenta) (representative of samples from 2 mice per group) or (B-D) dissociation for measurement of complement C3 deposition on lung cells using flow cytometry. (B) Gating strategy and representative histograms and quantification of median fluorescence intensity (MFI) of C3 on surfaces of lung endothelial cells (C) or leukocytes (D). Mean \pm SEM; horizontal lines are reference data from FMO controls. ${ }^{* *} P<0.001$ by 1 -way ANOVA and Dunnett's test vs. VE-Cad-Cre ${ }^{+}$anti- $-\mathrm{H}-2^{\mathrm{d}}-$ treated group, $n=6$.

endothelial MHC I removal on platelet and neutrophil responses. At 5 minutes after antibody injection, VE-Cad-Cre $\times \mathrm{B} 2 \mathrm{~m}^{\mathrm{f} / \mathrm{fl}}$ mice had reduced lung platelet retention as assessed by immunofluorescence and flow cytometry and a reduction in the severity of thrombocytopenia (Figure 4, A-D). However, anti-H-2 ${ }^{\mathrm{d}-\text { treat- }}$ ed mice lacking endothelial MHC I still became neutropenic, displayed normal neutrophil activation in lungs (surface CD11b upregulation), and had an exaggerated release of NETs at 5 minutes after anti-H-2 $2^{\mathrm{d}}$ treatment (Figure 4, E- $\mathrm{G}$ ). At 60 minutes after antibody injection, these mice had similar levels of lung neutrophil recruitment to those of control mice with intact endothelial MHC I (Figure 4H). These results indicate that endothelial MHC I ligation by antibody is important for lung platelet retention but dispensable for neutrophil responses.

Complement fixation onto pulmonary endothelial cells leads to lung platelet retention and edema in anti-MHC I-mediated lung injury. Complement deposition in lungs has been implicated in the induction of TRALI (8). Since mouse platelets lack Fc $\gamma$ receptors for direct antibody engagement, we hypothesized that complement fixation onto the pulmonary endothelium could lead to the retention of platelets and the development of pulmonary edema in the mouse TRALI model. Mice selectively deficient in endothelial MHC I (VE-Cad-Cre $\times$ B2 $\left.\mathrm{m}^{\mathrm{A} / \mathrm{f}}\right)$ showed deposition of $\mathrm{C} 3$ in their lungs at 5 minutes after anti- $\mathrm{H}-2^{\mathrm{d}}$ injection, but closer inspection and flow cytometric analysis of dissociated lung cells revealed that the $\mathrm{C} 3$ deposition was limited to leukocytes and largely absent on pulmonary endothelial cells in endothelial MHC I-deficient mice (Figure 5, A-D).

To establish a causal link between complement and lung platelet retention, we treated mice with cobra venom factor (CVF) to deplete complement through breakdown of $\mathrm{C} 3$ and $\mathrm{C} 5$. Mice treated with CVF showed reductions in plasma levels of C3 by more than $80 \%$ (Figure 6A) and had complete reductions in C3 fixation on lung endothelial cells and leukocytes when challenged with anti-H-2 ${ }^{\mathrm{d}}$ (Figure 6, B-D). CVF-treated mice did not develop lung platelet retention at 5 minutes after anti- $\mathrm{H}-2^{\mathrm{d}}$ administration and were protected from pulmonary vascular permeability, edema, and mortality 2 hours after anti-H-2 ${ }^{\mathrm{d}}$ injections (Figure 6, E-H). Interpreted together with our studies of endothelial MHC I-deficient mice, these findings suggest that complement activation on the endothelial surface is critical for anti-MHC I-mediated lung platelet retention, pulmonary edema, and mortality.

Human pulmonary endothelial HLA class I expression and complement activation in clinical TRALI. We hypothesized that 
BALB/c mice, LPS + cobra venom factor (CVF)/vehicle i.p., + $1 \mathrm{mg} / \mathrm{kg}$ anti-H-2d/isotype

A

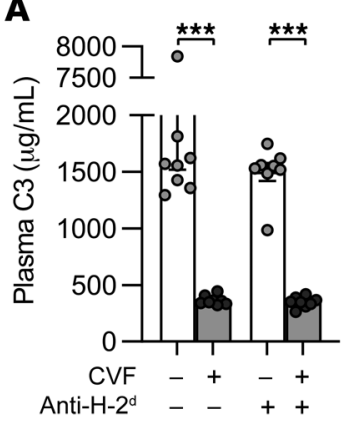

E

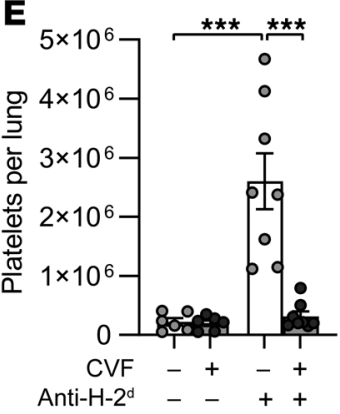

B

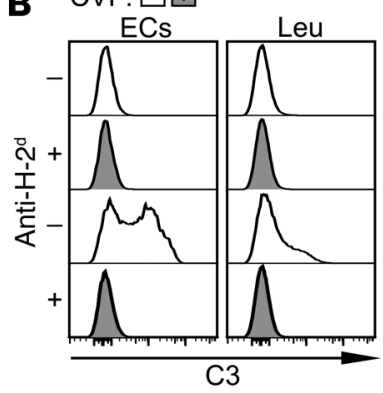

$\mathbf{F}$

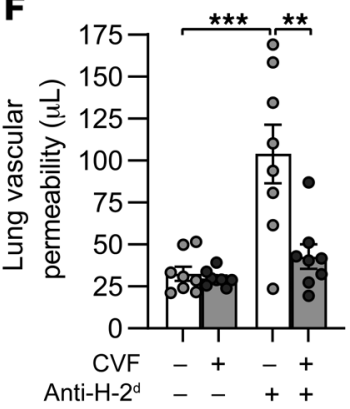

C

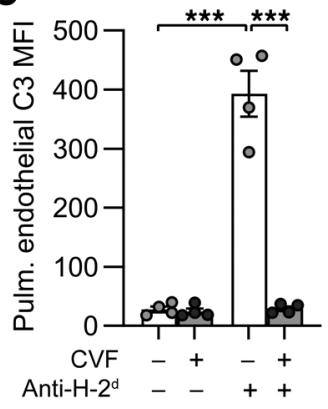

D

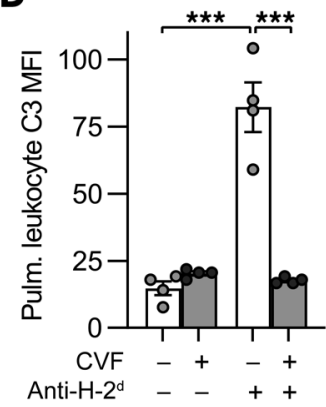

G

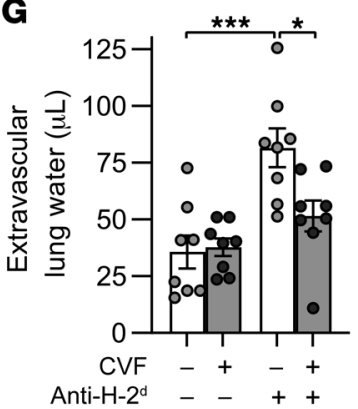

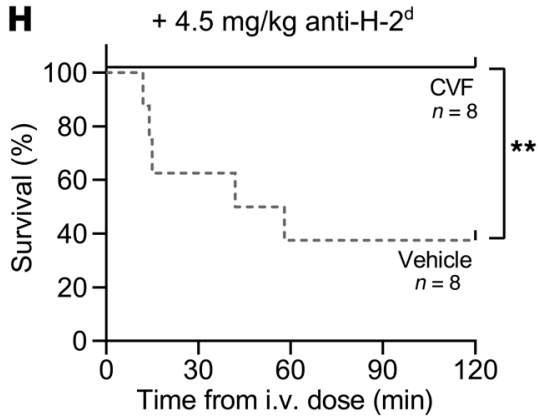

Figure 6. Complement depletion prevents anti-MHC I-mediated lung platelet retention, lung injury, and mortality. BALB/c mice were treated i.p. with LPS and either cobra venom factor (CVF) or vehicle, and 24 hours later were given i.v. injections of anti-H-2 $2^{\mathrm{d}}$ or isotype control antibody. (A) Plasma C3 concentrations, (B-D) cell surface C3 measurements from flow cytometric analysis of endothelial cells (ECs) or leukocytes (Leu) from dissociated lungs, and (E) platelet counts from dissociated lungs 5 minutes after antibody injections at $1 \mathrm{mg} / \mathrm{kg}$. (F and $\mathbf{G})$ Plasma extravasation and edema in lungs 2 hours after antibody injections at $1 \mathrm{mg} / \mathrm{kg}$, and $(\mathbf{H})$ survival after anti- $\mathrm{H}-2^{\mathrm{d}}$ antibody injections at $4.5 \mathrm{mg} / \mathrm{kg}$. Mean $\pm \mathrm{SEM}$. ${ }^{*} P<0.05 ;{ }^{* *} P<0.01 ;{ }^{* * *} P<0.001$ by 2-way ANOVA and Holm's test (A and $\mathbf{C}-\mathbf{G})$ or log-rank test (H). All $n=8$ with the exception of $\mathbf{C}$ and $\mathbf{D}$, where $n=4$.

if pulmonary endothelial antigen engagement and complement activation played a role in clinical TRALI, human pulmonary endothelial cells would have high surface HLA class I expression and complement activation would be detectable in blood plasma samples taken after development of TRALI. Reflecting the pattern observed in mice (Supplemental Figure 7, A-C), human pulmonary endothelial cells and monocytes showed high cell surface HLA class I expression, with lower expression on granulocytes and platelets (Figure 7, A-C). Classical/lectin pathway complement activation in humans can be detected through assessment of changes in $\mathrm{C} 4 \mathrm{~d}$ levels and the $\mathrm{C} 4 \mathrm{~d} / \mathrm{C} 4$ ratio (22). To test for the potential involvement of complement in human TRALI, we measured the $\mathrm{C} 4$ and $\mathrm{C} 4 \mathrm{~d}$ content of pre- and posttransfusion plasma samples from a study of patients receiving transfusions who either developed TRALI or did not (ref. 23 and Supplemental Table 1). Group comparisons showed that, accounting for baseline pretransfusion levels, plasma $\mathrm{C} 4 \mathrm{~d}$ levels and $\mathrm{C} 4 \mathrm{~d} / \mathrm{C} 4$ ratios were elevated after transfusion in patients who developed TRALI relative to transfused control patients who did not (Figure 7, D-G). One subject from the TRALI group showed a particularly high elevation in complement activation after transfusion, but an effect of TRALI status on complement activation was still detected even with this potential outlier removed (posttransfusion control vs. posttransfusion TRALI, Holm's tests: C4d, $P=0.043$; C4d/C4, $P=0.046)$. These results suggest that the human pulmonary endothelium is a feasible target for infused anti-HLA class I anti- bodies, and that complement activation occurs following transfusion in human TRALI cases.

Endothelial complement activation and antibody-mediated rejection of lung transplants. We also explored the role of anti-MHC antibodies and complement activation in a lung transplantation cohort. Anti-HLA donor-specific antibodies (DSAs) cause antibody-mediated rejection (AMR), which has emerged as an important cause of chronic lung allograft dysfunction (CLAD) or death in lung transplantation (4). It is plausible that mechanisms that drive complement-dependent AMR pathology are related to those that we identified in TRALI, as pulmonary microvascular endothelial complement C4d staining is used in AMR diagnosis and can be accompanied by histological and radiographic evidence of acute lung injury (Figure 8, A-C, and Supplemental Figure 11, A-C).

Anti-HLA de novo DSAs have previously been associated with increased risk of CLAD or death in lung transplantation (24-29), so we tested whether this was also the case in a cohort of 215 lung transplant recipients at UCSF (Supplemental Table 2). Of these patients, 44 tested positive for de novo DSAs against HLA class I or II. We found a $120 \%$ increased risk for CLAD or death in subjects who developed these DSAs at any time after lung transplantation relative to subjects without de novo DSAs (multivariable hazard ratio $[\mathrm{HR}] 2.2$, range $1.3-3.9, P=0.005)$. By treating the serologic detection of DSAs as a time-dependent variable, the persistence of DSAs also conferred an increased risk of CLAD or death (Figure 8D; multivariable HR 1.5, range 1.1-1.9, $P=0.003$ ). Both anti-HLA 
Human lung cells

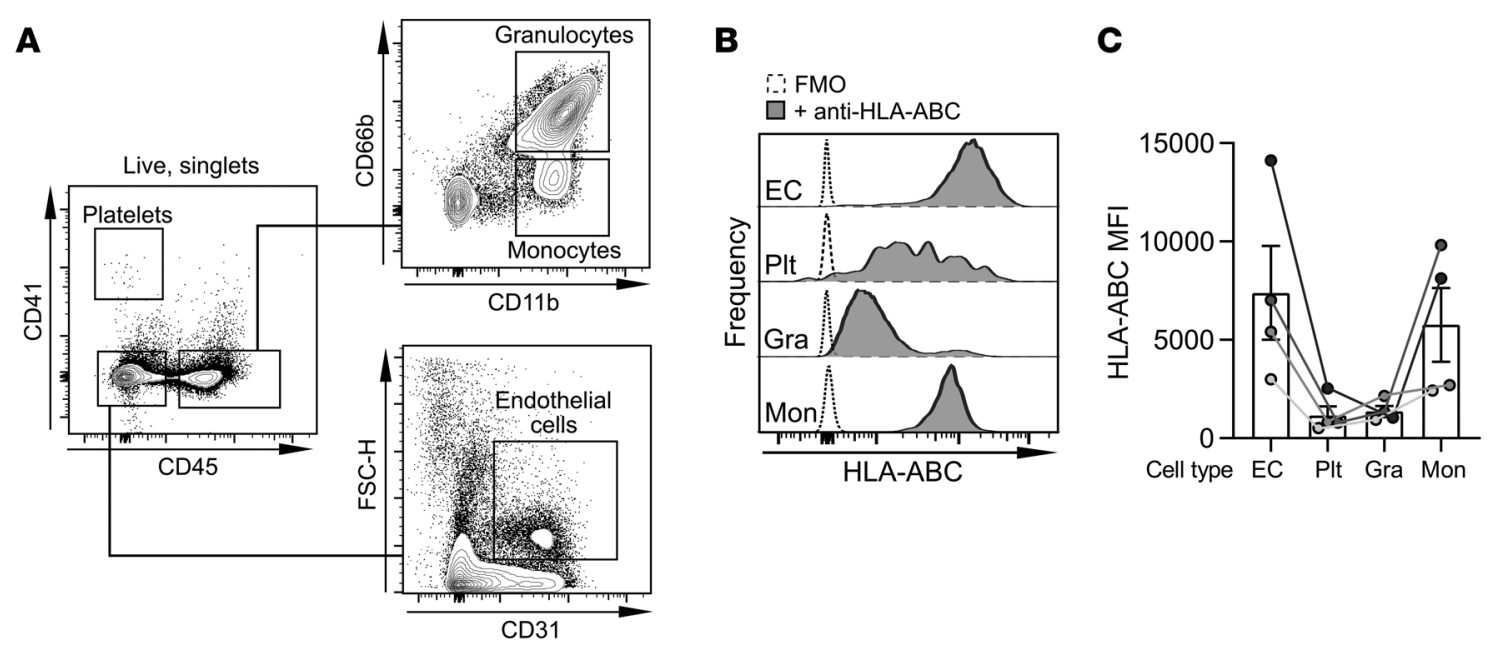

Human blood plasma

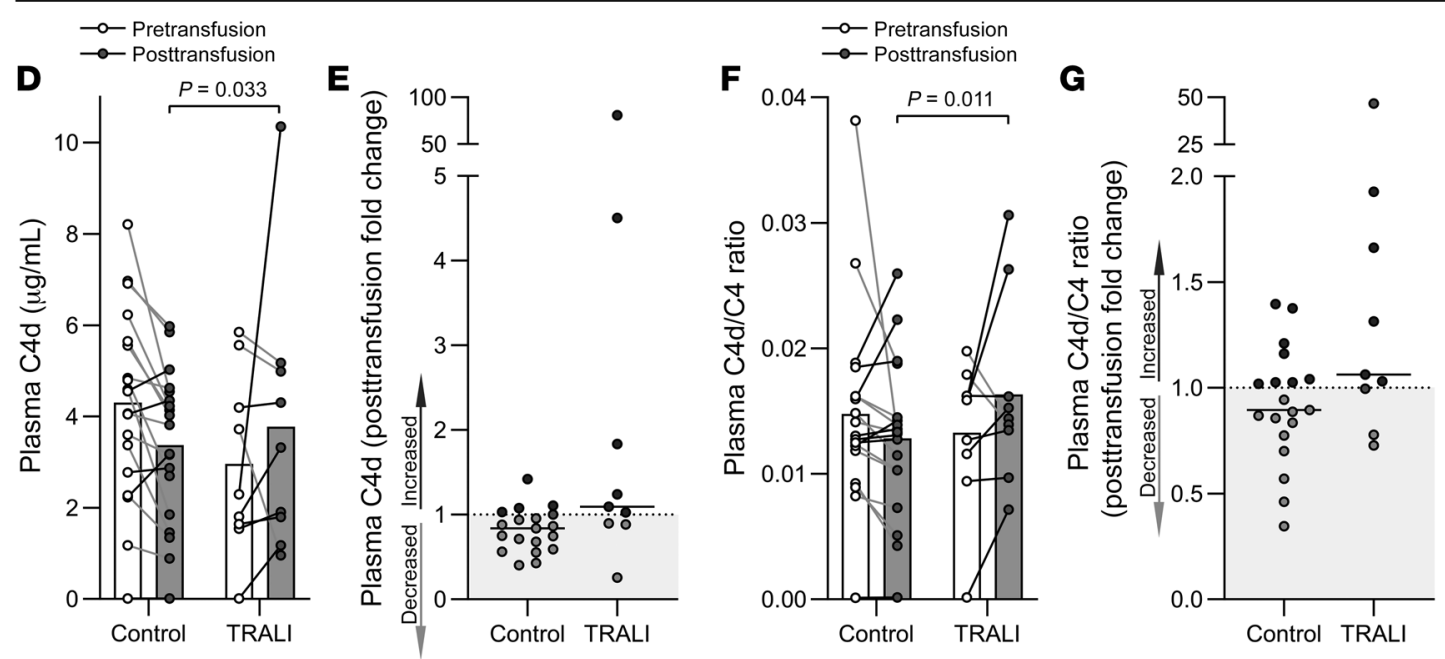

Figure 7. High endothelial HLA class I expression in human lungs and complement activation after transfusion in clinical TRALI. Samples of human lungs were dissociated for measurement of cell surface HLA class I (HLA-ABC) expression using flow cytometry. (A) Cating strategy, (B) representative histograms of fully stained cells relative to FMO controls, and (C) quantification of HLA class I expression on different cells expressed as MFI (D-G) ( $n=4)$. EC, endothelial cell. Plasma content of the classical/lectin pathway complement component C4 and its activation and degradation product C4d were measured using pre- and posttransfusion samples from patients diagnosed with TRALI or control patients who did not develop TRALI after transfusion. (D) Concentrations of C4d and (E) fold change in C4d after transfusion, and (F) C4d/C4 ratios and (C) fold change in C4d/C4 ratios after transfusion. Normalized data (E and $\mathbf{G}$ ) provided for enhanced display of individual changes after transfusion. Mean ( $\mathbf{D}$ and $\mathbf{F}$, bars) or median (E and $\mathbf{G}$, lines) and individual values ( $\mathbf{D}$ and $\mathbf{F}$ ). In $\mathbf{D}$ and $\mathbf{F}$, a 2-way repeated-measures mixed-model approach was used with pretransfusion values set as covariates, followed by Holm's multiplicity correction for comparisons of effect of TRALI development after transfusion and effect of transfusion within the control group and the TRALI group. Control group, $n=19$; TRALI group, $n=9$. $P$ values $<0.05$ are shown .

class I and anti-HLA class II DSAs were associated with greater risk of CLAD or death (class I: multivariable HR 1.4, range 1.0-1.9, $P=0.044$; class II: multivariable HR 1.7, range 1.2-2.2, $P<0.001$, and the presence of antibodies against both HLA classes appeared to have an additive effect (Figure 8E; multivariable HR 2.9, range 1.2-7.1, $P=0.02$ ). These observations add to the evidence that anti-HLA DSAs, which can activate complement on the pulmonary endothelium, are harmful in lung transplantation.

Complement assists anti-MHC I-activated neutrophils to release NETs. Lung neutrophils still became activated (upregulating surface CD11b) in CVF-treated mice following anti-H-2d injections, indicating a complement-independent (i.e., Fc $\gamma$ receptor-mediat- ed; refs. 8, 12) pathway to neutrophil activation (Figure 9A). However, in marked contrast to endothelial MHC I-deficient mice, complement-depleted mice showed complete reductions in intravascular NETs (Figure 9B). NET formation was live imaged in antibody-injured lungs using membrane-impermeant SYTOX Green DNA dye injected i.v. (30), which revealed swollen cells with compromised membranes, filamentous strands of intravascular NETs, and abundant particles of extracellular DNA within minutes of anti-H-2d injection (Figure 9C and Supplemental Video 4).

As release of NETs into blood was present and enhanced in the absence of endothelial MHC I (Figure 4G), we hypothesized that antibody binding to blood cell MHC I was sufficient to acti- 
Antibody-mediated rejection
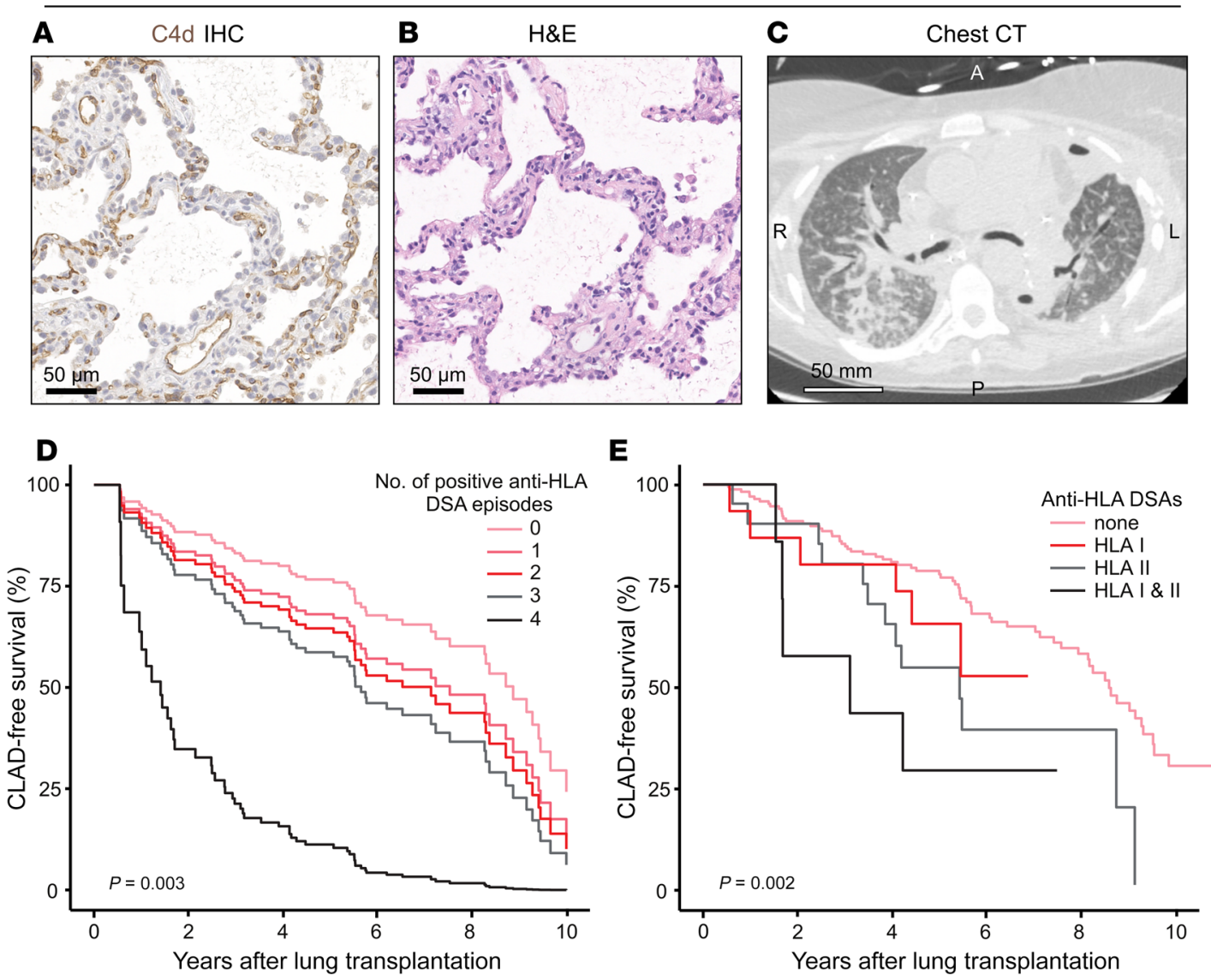

Figure 8. Anti-HLA antibodies in lung transplant recipients activate complement on pulmonary endothelium and increase risk of CLAD or death. Serial sections of a transbronchial biopsy from a lung transplant recipient diagnosed with antibody-mediated rejection (AMR) showing (A) diffuse, strong linear microvascular endothelial C4d positivity (immunohistochemistry: C4d = brown, hematoxylin counterstain = blue), and (B) organizing acute lung injury with increased neutrophils (H\&E staining). (C) Chest computed tomography (CT) scan from lung transplant recipient diagnosed with AMR showing bilateral consolidations. ( $\mathbf{D}$ and $\mathbf{E}$ ) Kaplan-Meier curves showing chronic lung allograft dysfunction-free (CLAD-free) survival over time in lung transplant recipients comparing effects of (D) number of episodes of anti-HLA donor-specific antibodies (DSAs) (adjusted curves from Cox proportional hazards model using serologic detection of DSAs as a time-dependent variable), or (E) different reactivities of de novo anti-HLA DSAs (actual CLAD-free survival within groups). (D and E) $P$ values are from log-rank tests from whole Cox models, $n=215$ with 15 patients with anti-HLA class I DSAs only, 22 with anti-HLA class II DSAs only, and 7 with detected DSAs against both HLA classes.

vate neutrophils and elicit NETs. In EDTA-anticoagulated B6.H2 mouse blood incubated with antibodies for 5 minutes, neutrophils displayed anti-H- ${ }^{\mathrm{d}}$ dose-dependent activation, upregulating surface CD11b, and shedding CD62L (Figure 9, D-G).

EDTA inhibits complement activation, whereas PPACK, an anticoagulant that inhibits thrombin and the contact system, spares extracellular $\mathrm{Ca}^{2+}$ and $\mathrm{Mg}^{2+}$ to permit complement cascade activation. With PPACK anticoagulation, anti- $\mathrm{H}-2^{\mathrm{d}}$ antibody caused a greater proportion of neutrophils to take up membrane-impermeant DAPI DNA dye, indicating membrane pore formation (Figure 9, H-J). Incubation of PPACK-anticoagulated blood and antibody with the fixable membrane-impermeant DNA dye, DRAQ7, enabled immunofluorescence confirmation of anti-H-2 ${ }^{\mathrm{d}}$-induced neutrophil membrane permeabilization, nuclear swelling, and DNA release (Figure 9, K and L). The release of in vitro-elicited NETs into plasma could also be detected by ELISA (Figure 9M). Finally, CVF pretreatment of mice reduced the in vitro release of NETs into plasma following incu- bation with anti-H-2 ${ }^{\mathrm{d}}$ (Figure $9 \mathrm{~N}$ ). These data suggest that local complement activation can act to drive antibody-activated neutrophils toward NET formation, which is a critical pathophysiologic mechanism in TRALI.

\section{Discussion}

The major findings of this study were that (a) antibody engagement of endothelial antigen is necessary and sufficient for initiation of anti-MHC I-mediated lung injury, (b) complement activation is critical for both pulmonary edema and NET release downstream of antibody binding, and (c) lungs can tolerate massive antibodyinduced complement activation, neutrophil retention, and NET release without lethal edema when the endothelium is spared from antibody and complement binding. In the course of these studies we also developed a B6-congenic approach to modeling TRALI in mice, a mouse model permitting conditional restoration of MHC I expression, and an assay for complement-driven NET release in whole blood. These methods may be useful for the growing num- 
In vivo
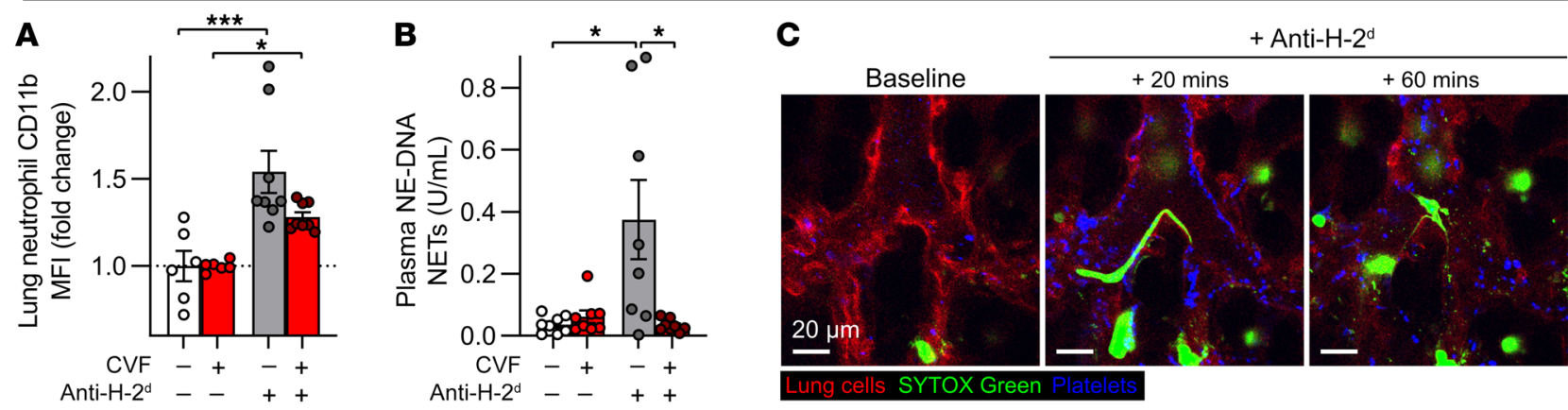

In vitro
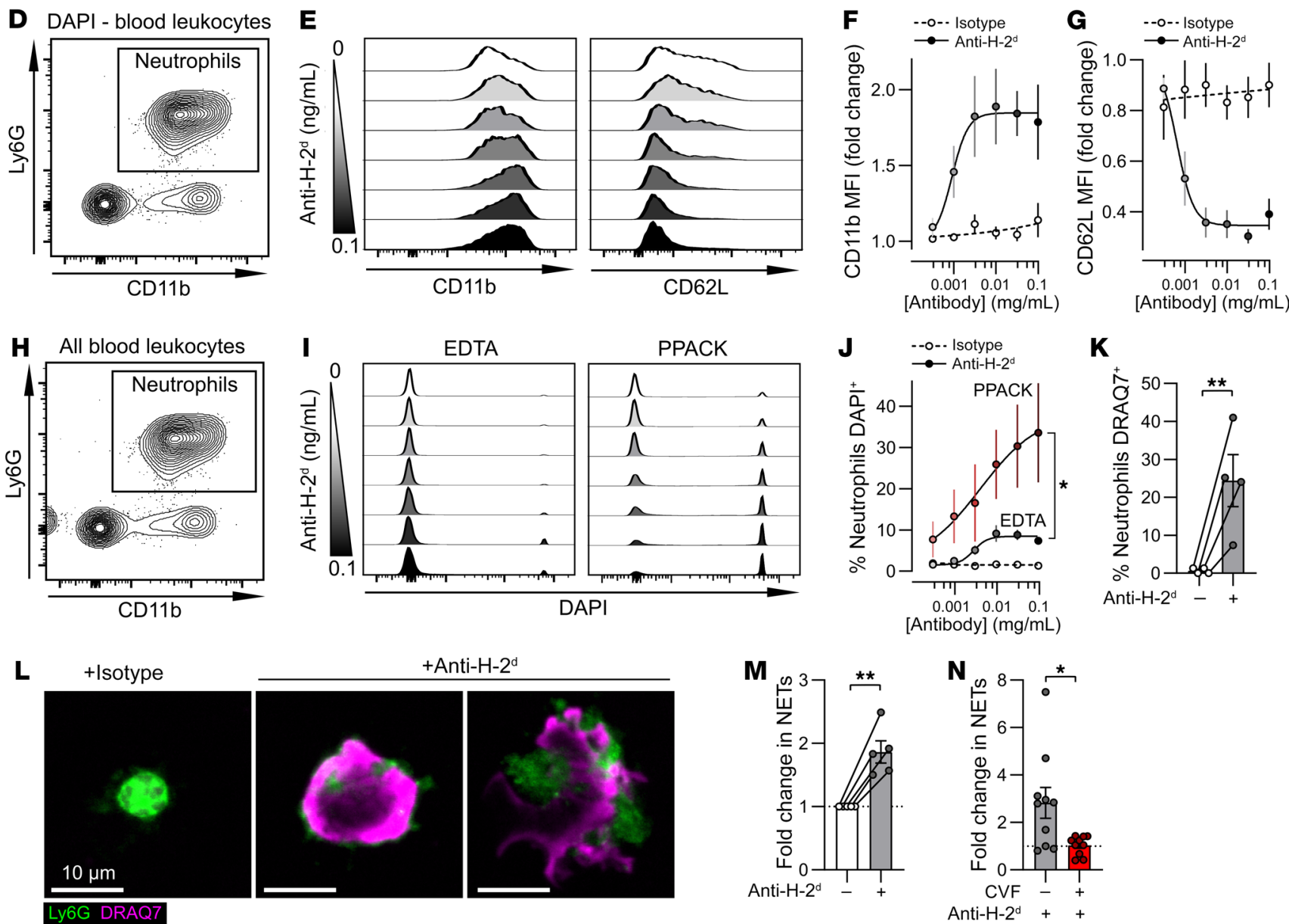

Figure 9. Anti-MHC I-mediated release of NETs into blood requires complement activation. Effect of cobra venom factor (CVF) pretreatment and anti-H-2 $2^{\mathrm{d}}$ injections (1 mg/kg i.v., +5 minutes) on (A) lung neutrophil surface CD11b expression and (B) release of neutrophil elastase (NE)-DNA complexes (NETs) into plasma in LPS-primed BALB/c mice $(n=8)$. (C) Intravital lung imaging of release of NETs following anti-H-2 ${ }^{\mathrm{d}}$ antibody injection into LPSprimed B6. $2^{d} \times$ PF4-Cre $\times$ mTmG mice (cells in lungs, red; SYTOX Green-dyed DNA, green; platelets, blue) (representative of imaging from 6 mice). (D-J) Blood was collected from LPS-primed B6. $2^{d}$ mice, EDTA anticoagulated, and stimulated with anti-H-2d/isotype for 5 minutes before flow cytometric measurements of DAPI- neutrophil $(\mathbf{E}$ and $\mathbf{F})$ CD11b and $(\mathbf{G}$ and $\mathbf{H})$ CD62L surface expression $(n=4)$. $(\mathbf{H}-\mathbf{J})$ Blood was collected from LPS-primed B6. H2 ${ }^{d}$ mice, anticoagulated with EDTA or PPACK, and uptake of DAPI by neutrophils was assessed using flow cytometry following 5 minutes of anti-H-2 $/$ isotype stimulation $(n=4)$. (K-M) Alternatively, PPACK-anticoagulated blood was used for ( $\mathbf{K}$ and $\mathbf{L}$ ) imaging of neutrophil uptake of DRAQ7 (neutrophil Ly6G, green; DRAQ7-DNA, magenta), and (M) ELISA measurements of release of NETs into plasma following 60 minutes of $0.01 \mathrm{mg} / \mathrm{mL}$ anti-H-2 ${ }^{\mathrm{d}}$ stimulation (normalized to isotype controls). (N) Separate B6.H2 ${ }^{d}$ mice were given i.p. LPS and either CVF or vehicle, and blood plasma was collected for ELISA measurements of NE-DNA NETs following 60 minutes of $0.01 \mathrm{mg} / \mathrm{mL}$ anti-H-2 ${ }^{\mathrm{d}}$ stimulation (normalized to isotype controls) $(n=10)$. Mean $\pm \mathrm{SEM}$. ${ }^{*} P<0.05 ;{ }^{* *} P<0.01$; ${ }^{* *} P<0.001$ by 2 -way ANOVA with Holm's test ( $\mathbf{A}$ and $\left.\mathbf{B}\right), 1$-way ANOVA main effect of anticoagulant (J), paired $t$ test (K and $\left.\mathbf{M}\right)$, or unpaired $t$ test $(\mathbf{N})$. 
ber of research groups using mouse TRALI models, researching HLA/MHC I immunology, or studying the release of NETs.

Our results have potential implications beyond anti-HLA class I-mediated TRALI. In addition to HLA/MHC class I, endothelial cells also express other antigens implicated in TRALI, including human neutrophil antigen-3a/CTL2 (31) and HLA/MHC class II (32), so it is possible that endothelium-directed complement activation is a central mechanism driving antibody-mediated TRALI.

Associations between de novo anti-HLA DSAs and reductions in CLAD-free survival that we identified in the cohort of lung transplant patients are also further evidence that antibodies are mediators of lung injury in this context. Increasing evidence suggests that complement is a mediator of graft injury in antibody-mediated lung transplant rejection $(33,34)$, and the role of NETs and the potential utility of complement inhibition are subjects of ongoing investigations in $\operatorname{AMR}(4,35)$. Adapted mouse TRALI models may be useful for proof-of-concept studies for potential AMR therapeutics.

NETs are important mediators of antibody-induced lung injury $(36,37)$, can directly cause endothelial damage (36), and can induce alternative pathway complement activation (38). Here we also found that, similar to the induction of NETs by some bacterial strains (39), complement activation is a required step for neutrophils to release NETs in response to antibody engagement of host cells. This requirement may help to prevent potentially harmful NET formation when antibodies bind cells but do not also sufficiently activate complement, and might be mediated via the CD35 or CD11b complement receptors (39), or through terminal complement activation on neutrophil surfaces leading to membrane pore formation, analogous to the role previously described for gasdermin D pore formation (40). A requirement for complement activation also explains previous observations that the neutrophil reactive oxygen species burst was not induced by anti-H-2 $2^{\mathrm{d}}$ antibody using washed, bone marrow-derived neutrophils in complement-free media (12).

Recent studies have indicated that the pulmonary vasculature may be an important site for depriming of activated neutrophils (41). Our results demonstrate that substantial retention of activated neutrophils and release of NETs can occur in the absence of major lung injury so long as endothelium-directed complement activation is absent. This observation is consistent with our previous finding that DNase I treatment increased NETs in plasma but protected against injury (36), suggesting that increases in levels of circulating NETs alone is not sufficient for lung injury in this model. At present, it is unknown to what extent pulmonary edema in TRALI results from a direct endothelium-intrinsic response to fixation with complement, or whether excessive leak is primarily a result of neutrophil or monocyte activation by, or phagocytosis of, pulmonary endothelial cells tagged with antibody and complement (42). A deeper understanding of the essential complement components, signaling pathways, and effector mechanisms will be important for targeting of strategies to prevent antibody-induced pulmonary edema, and for understanding how the lung protects the rest of the body from primed neutrophils without sustaining injury.

Our measurements of high pulmonary endothelial MHC I expression in human lungs, elevated posttransfusion complement activation in TRALI cases, and associations of anti-HLA DSAs with reduced CLAD-free survival in lung transplantation are sugges- tive that the mechanisms we identified as causal in murine TRALI may also occur in humans. However, there are many differences between the immune systems of mice and humans, and the role that complement activation plays in clinical TRALI and AMR is incompletely understood $(4,43)$. Our study of C4d levels in TRALI patient plasma was limited by the small number of available samples, the indirect approach of measuring residual C4d and C4 in blood plasma, and the heterogeneous nature of human TRALI (1). Blood plasma C4d levels were high in some patients who developed TRALI and some controls who did not, likely reflective of the hospitalized health status of this patient cohort (ref. 23 and Supplemental Table 1). Nevertheless, our data are consistent with posttransfusion C4d generation as a feature of at least some clinical TRALI cases.

The susceptibility of mice with MHC I expression exclusively on endothelium to experimental TRALI confirms the importance of endothelial antigen engagement in this model. This finding is consistent with the conclusions of a previous bone marrow transfer mouse study that implicated nonhematopoietic MHC I in TRALI initiation (8), and avoids potential confounding factors of the approach of this prior study (irradiation, transfer of MHC-mismatched hematopoietic cells, and anti- $\mathrm{H}-2^{\mathrm{d}}$ reactivity with $\mathrm{H}-2 \mathrm{~K}^{\mathrm{b}}$ as well as $\mathrm{H}-2^{\mathrm{d}}$ antigens). Among the hematopoietic cell types that we studied, our most notable results were that monocytes showed particularly high surface MHC I expression, and removal of Ly6C ${ }^{\text {lo }}$ monocyte MHC I increased antibody-induced pulmonary edema. It is therefore possible that this large pool of antigen on monocytes may act as a sink for antibody that would cause additional harm were it to bind endothelial antigen. Alternatively, removal of Ly6 $\mathrm{C}^{\text {lo }}$ monocyte MHC I might have exacerbated edema by sparing injurypromoting monocytes from antibody-mediated clearance $(8,44)$.

Platelets are implicated as effectors of lung injury in TRALI $(36,45-47)$, although the role of platelets in promoting antibody-mediated injury has been controversial $(8,48)$. Intravital lung imaging revealed platelet adhesion to pulmonary endothelium and occasional thrombotic events. The complement dependence of recruitment of platelets to antibody-tagged endothelium explains the rapid reaction of platelets in mice where platelet $\mathrm{Fc} \gamma$ receptors are absent, and thrombotic responses to antibody may be increased in human platelets with Fc $\gamma$ RIIA expression (49). There are many pathways through which platelets might become adhesive in response to complement activation on endothelium: through platelet receptors for complement $\left(\alpha_{\mathrm{Ib}} \beta_{3}-\right.$ factor $\mathrm{H}$ [ref. 50], P selectin-C3b [ref. 51], C3aR-C3a [ref. 52]), or through endothelial cell exocytosis or exposure of the basement membrane during inter-endothelial cell gap formation. Together with previous findings of reductions in lung injury with platelet depletion or $\alpha_{\mathrm{II}} \beta_{3}$ inhibition $(15,36,45)$, observations of platelet thrombi and loss of perfusion in TRALI lungs point toward a scenario where pulmonary thrombosis could impair gas exchange through limiting lung microvascular perfusion - especially where there is thrombus involvement of activated neutrophils and NETs (53). Additionally, platelets can amplify complement activation, providing a mechanistic link between platelet activation, NET release, and endothelial permeability $(36,51)$. Aspects of platelet function may also be protective against bleeding in TRALI (48), so it will be important to determine whether platelet recruitment to endothelium observed here in experimental TRALI, and previous- 
ly following LPS inhalation (54), or the AMR of skin grafts (55), is a protective or harmful response.

In summary, our findings suggest that TRALI resulting from anti-MHC/HLA antibodies is secondary to antibody binding to these antigens on endothelial cells. Responses dependent on endothelial antigen (pulmonary edema and platelet retention) and independent of presence of endothelial antigen (release of NETs) shared a dependence on complement activation, so targeting complement activation may therefore be useful in TRALI and in other settings where endothelial injury, microvascular platelet retention, and the release of NETs make contributions to pathology.

\section{Methods}

Mice. Mice were housed in the UCSF Laboratory Animal Research Center specific pathogen-free facility. BALB/c mice (Charles River) and C57BL/6 mice (Jackson Laboratory) were housed in the UCSF vivarium for 2 or more weeks before use. Other strains were both bred and housed at UCSF (see Supplemental Table 3 for origins), genotyped using PCR, and were used at age 6 to 12 weeks, with male mice at 8 to 12 weeks used for lung injury experiments. The $\mathrm{B} 2 \mathrm{~m}^{\text {inv }}$ strain was created by James C. Zimring (University of Virginia, Charlottesville) using homologous recombination to modify the $B 2 m$ locus in embryonic stem cells of the $\mathrm{B} 6$ genetic background (Bruce- 4 cell line). Mice were generated from neomycin-resistant clones with the desired recombination (as confirmed by Southern blot); the NEO cassette was flanked with FRT sites and removed through exposure to FLP recombinase (omitted in simplified diagrammatic representation of $B 2 \mathrm{~m}$ locus). Exons 2 and 3 of B2m were inverted and flanked with loxP and lox2272 variants such that exposure to Cre would result in an irreversible reversion of the exon (56). The resulting locus was predicted to not express B2m unless it encountered Cre recombinase, resulting in reversion of exons 2 and 3 and a normal open reading frame for translation of $\mathrm{B} 2 \mathrm{~m}$ (Figure $2 \mathrm{~A})$.

TRALI model. Mice were primed with LPS (O55:B5 serotype, MilliporeSigma, $0.1 \mathrm{mg} / \mathrm{kg}$, i.p.) 24 hours before injections of anti-H-2 ${ }^{\mathrm{d}}$ antibody or isotype (BE0180 clone 34-1-2S or BE0085 clone C1.18.4, Bio X Cell, given at 1 or $4.5 \mathrm{mg} / \mathrm{kg}$, i.v. where indicated). Antibody infusions were carried out under ketamine/xylazine anesthesia $(0.6 / 0.1 \mathrm{mg} / \mathrm{kg}$, i.p.) with samples collected at indicated time points. For measurements of lung extravascular plasma equivalents (lung vascular permeability) and bloodless extravascular lung water, ${ }^{125} \mathrm{I}$-albu$\min (0.1 \mathrm{MBq} / \mathrm{kg}$, i.v., Iso-Tex) was injected together with antibody, then at 2 hours later or at cessation of respiratory movements blood was collected via cardiac puncture and lungs were excised for quantification of radioactivity (Packard 5000 series) and gravimetric analysis, as previously described $(12,15,36)$.

Flow cytometry. Blood $(50 \mu \mathrm{L})$ was sampled via submandibular vein or inferior vena cava, red cells lysed (red cell lysis buffer: $53 \mathrm{mM}$ $\mathrm{NHCl}_{4}, 10 \mathrm{mM} \mathrm{KHCO}, 0.1 \mathrm{mM} \mathrm{EDTA}$ ), and platelets and leukocytes resuspended in FACS buffer (PBS, 0.3\% BSA, 0.5 mM EDTA). Mouse lungs were excised en bloc, or $10-\mathrm{mm}^{3}$ segments were sampled from the inferior right lower lobe of human donor lungs that did not meet clinical criteria for transplantation utilization (Donor Network West). Lungs were kept on ice and subsequently diced into pieces less than 2 $\mathrm{mm}$ in diameter in PBS with $1 \mathrm{mg}$ Liberase (Roche) and 100 U DNase I (Roche). Samples were then incubated at $37^{\circ} \mathrm{C}$ for 2 hours before passing through a $40-\mu \mathrm{m}$ filter, red cell lysis, and resuspension of one-fifth of total lung cells in FACS buffer plus Fc block (mouse: clone 2.4G2, UCSF Monoclonal Antibody Core; human: BD Fc Block, catalog 564219 ; both $1 \mu \mathrm{g} / \mathrm{mL}$ ) for staining. Fluorophore-tagged antibodies (See Supplemental Table 4) were incubated with samples at $4^{\circ} \mathrm{C}$ for 30 minutes. For absolute cell counts, quantification beads (CountBright, Thermo Fisher Scientific) were used. Samples were analyzed using an LSRII flow cytometer (BD) with gating and measurements using FlowJo software (BD) (representative full gating strategies are shown in Supplemental Figure 12).

Intravital microscopy. Two-photon intravital lung microscopy was performed as previously described $(57,58)$. Following anesthesia with ketamine/xylazine (0.6/0.1 mg/kg i.p.), the right jugular vein was cannulated and kept patent with $150 \mu \mathrm{L} /$ hour normal saline for i.v. dosing (Instech), before ventilation with room air plus 1\% isoflurane at 125 breaths/min at $10 \mu \mathrm{L} / \mathrm{g}$ body weight breath volume (Minivent, Harvard Apparatus), with $2-3 \mathrm{cmH}_{2} \mathrm{O}$ positive end-expiratory pressure. A thoracic window was then inserted into an intercostal incision and a section of the left lung was immobilized against the window with $25-30 \mathrm{mmHg}$ negative pressure. Imaging experiments used a Nikon A1r (UCSF Biological Imaging Development Center [BIDC]) with a CFI75 Apochromat 25XC W 1300 objective (Nikon), scanning with a $950 \mathrm{~nm}$ laser line from a Mai Tai DeepSee Ti:sapphire laser, with emitted light collected through 400-492, 500-550, 563-588, and 601-657 $\mathrm{nm}$ bandpass filters. Where indicated, FITC-dextran (70 kDa, $50 \mathrm{mg} / \mathrm{kg})$, Evans blue ( $3 \mathrm{mg} / \mathrm{kg}$ ), or SYTOX Green (0.6 mg/kg; Thermo Fisher Scientific) was given i.v. before imaging. Image processing and analysis was performed using Imaris (Bitplane), using sum fluorescence intensities of fluorophores for quantification with MATLAB channel arithmetic (MathWorks) used to subtract fluorophore spillover where required.

Immunofluorescence. Lungs were fixed by inflation and immersion in $1 \%$ formaldehyde for 2 hours, cryoprotected with $30 \%$ sucrose overnight, and frozen in OCT. Cryosections $(100 \mu \mathrm{m})$ were stained with primary antibodies in PBS containing 0.3\% Triton X-100, 0.2\% BSA, and $10 \%$ secondary host serum overnight, washed, and incubated with secondary antibodies in PBS with 0.3\% Triton X-100 (antibody details in Supplemental Table 4), before a final wash. Blood smears were fixed with $4 \%$ formaldehyde for 10 minutes, blocked with PBS plus $1 \%$ BSA for 15 minutes, washed, and incubated with labeled antibody in PBS plus 1\% BSA for 2 hours before a final wash. Confocal imaging was carried out using a Nikon A1r (UCSF BIDC) with a CF175 LWD $16 \times$ W objective.

Blood counts. Blood was collected via cardiac puncture into K2EDTA MiniCollect tubes (Greiner) for complete blood counts using a Genesis analyzer (Oxford Science).

Histology. Mouse lungs were fixed by inflation and immersion in $4 \%$ formaldehyde for more than 24 hours. Mouse lungs and human transbronchial biopsies were processed, paraffin wax-embedded, sectioned at $4 \mu \mathrm{m}$, and stained with hematoxylin and eosin (H\&E) for analysis using standard methods.

Complement depletion. Immediately after LPS priming, at -24 hours before antibody injections, mice were injected with CVF $(0.4 \mathrm{mg} / \mathrm{kg}$ i.p., 233552 from Naja naja kaouthia, MilliporeSigma) or PBS vehicle.

NET ELISA. Neutrophil elastase-DNA complexes were quantified using anti-neutrophil elastase capture antibody (sc-55549, G-2, Santa Cruz Biotechnology) coated 1:250 onto a MaxiSorp plate (Nunc), with peroxidase-conjugated anti-DNA reporter antibody (MCA-33, Roche) followed by tetramethylbenzidine development (R\&D Systems) 
according to the manufacturers' instructions, as previously reported $(30,59)$. NET units were calculated relative to serial dilution calibration curves of BAL supernatant from mice 9 days after i.n. dosing with 50 PFU of influenza A virus (A/PR/8/34 H1N1, $1 \mathrm{U}=$ bioequivalence).

Complement ELISAs. Pretransfusion and posttransfusion plasma samples were tested from transfused controls and TRALI subjects from the UCSF arm of the TRALI case-control study (23). ELISAs for mouse C3 (ab157711, Abcam), human C4 (ab108824, Abcam), and human C4d (A009, Quidel) were used according to manufacturers' instructions.

Clinical lung transplant studies. Adults who received single lung, bilateral lung, or heart-lung allografts at UCSF between December 1, 2006 and August 16, 2018 were included if they survived to the first follow-up lung function study (approximately 14 days), had at least 1 donor-specific antibody measurement, and provided informed consent. CLAD was defined according to established criteria as an unresolving $20 \%$ decline in $\mathrm{FEV}_{1}$ or FVC lasting over 30 days (60), and adjudicated as previously described $(61,62)$. CLAD-free survival was quantified as days after transplantation of freedom from CLAD or death. Standard posttransplant induction regimens for all subjects included methylprednisolone, mycophenolate, and basiliximab. Maintenance immunosuppressant therapy included tacrolimus, prednisone, and mycophenolate mofetil. DSAs were measured on a Luminex-based platform as part of routine clinical care according to established protocols (29). Positivity for DSAs was defined as mean fluorescence intensity greater than 2,000. Persistently positive DSAs were treated with monthly infusions of i.v. immunoglobulin.

In vitro neutrophil assays. Mice were anesthetized with isoflurane and blood was collected from the inferior vena cava, anticoagulated with either EDTA (2 mg/mL, K2EDTA MiniCollect, Greiner) or PPACK (80 $\mu \mathrm{M}, H$-D-Phe-Pro-Arg-chloromethylketone trifluoroacetate, Bachem) and incubated with antibodies at $37^{\circ} \mathrm{C}$ for indicated times. At stated time points, blood was either RBC-lysed and cooled to $4^{\circ} \mathrm{C}$ for flow cytometry experiments, immediately used for blood smears, or transferred to a K2EDTA tube for separation of plasma.

Statistics. For genetic experiments, mice were assigned numerical IDs at the time of genotyping, which were used for blinded identification during data collection. $\mathrm{CVF} / \mathrm{anti}-\mathrm{H}-2^{\mathrm{d}}$ treatments were assigned unblinded using within-cage blocked randomization, with survival analysis carried out blinded to treatment group. Residuals versus predicted plots were used to assess requirement for transformations and for identification of outliers, and violations of proportional hazards were assessed visually and with the Schoenfeld test. For data from lung transplant recipients, we used Cox proportional hazards models to determine CLAD-free survival HRs as a function of DSA episodes and antigen, which included covariates for subject characteristics frequently associated with poor transplant outcomes: age at transplantation, recipient sex, recipient reported ethnicity, transplant diagnosis, and transplant type (single, double, or heart-lung). Additional analyses were also adjusted for the time dependence of DSA measurements. Statistical tests were applied as per figure legends with $P$ less than 0.05 as the significance threshold. All $t$ tests were 2-tailed. GraphPad Prism, InVivoStat, and R (using the "survminer," "plyr," "ggplot," "survival," "stringr," and "ggpubr" packages) were used for data analysis and graphing.

Study approval. All animal experiments were approved by the Institutional Animal Care and Use Committee at UCSF. All human subjects were enrolled in a protocol approved by the UCSF Committee for Human Research (IRB).

\section{Author contributions}

SJC, NK, and MRL designed the experiments. SJC, NK, JJT, DRC, $\mathrm{BM}, \mathrm{MM}$, and MRL conducted experiments and acquired data. CAL, CNM, AMH, HLH, and JCZ provided mice. MM, PT, AU, and CAL provided reagents and samples. DRC, JRG, JPS, SRH, and JK conducted the UCSF lung transplant clinical study. SJC and DRC performed statistical analysis, SJC and MRL wrote the original draft of the manuscript. All authors made contributions to reviewing and editing the manuscript.

\section{Acknowledgments}

We acknowledge the UCSF BIDC for enabling imaging experiments, and the UCSF Parnassus Flow Core, supported in part by the NIH Diabetes Research Center grant P30 DK063720, for flow cytometry support. We thank Michael Matthay (UCSF, San Francisco), Donor Network West, the TRALI study group, and participating donors and clinicians for providing access to human samples, and Kirk D. Jones (UCSF, San Francisco) for providing lung AMR C4d images. SJC would like to thank Peter Baluk and Donald McDonald for training in immunofluorescence techniques, Emma Lefrançais for intravital training, and Clare Abram and Alexandre Boissonnas for insights into myeloid cell biology. This work was supported by NIH R01 grants HL107386 (to MRL), HL130324 (to MRL), HL138673 (to MRL and JCZ), and an International Society for Heart and Lung Transplant Joel D. Cooper Award (to DRC).

Address correspondence to: Mark R. Looney, 513 Parnassus Avenue, HSE 1355A, San Francisco, California 94143-0130, USA. Phone: 415.476.9563; Email: mark.looney@ucsf.edu.
1. Semple JW, Rebetz J, Kapur R. Transfusion-associated circulatory overload and transfusion-related acute lung injury. Blood. 2019;133(17):1840-1853.

2. Vossoughi S, et al. Ten years of TRALI mitigation: measuring our progress. Transfusion. 2019;59(8):2567-2574.

3. Vlaar APJ, et al. A consensus redefinition of transfusion-related acute lung injury. Transfusion. 2019;59(7):2465-2476.

4. Kulkarni HS, Bemiss BC, Hachem RR. Antibody-mediated rejection in lung transplantation. Curr Transplant Rep. 2015;2(4):316-323.

5. Popovsky MA, Abel MD, Moore SB. Transfu- sion-related acute lung injury associated with passive transfer of antileukocyte antibodies. Am Rev Respir Dis. 1983;128(1):185-189.

6. Sachs UJ, et al. Mechanism of transfusion-related acute lung injury induced by HLA class II antibodies. Blood. 2011;117(2):669-677.

7. Dykes A, Smallwood D, Kotsimbos T, Street A. Transfusion-related acute lung injury (Trali) in a patient with a single lung transplant. Br J Haematol. 2000;109(3):674-676.

8. Strait RT, et al. MHC class I-specific antibody binding to nonhematopoietic cells drives complement activation to induce transfusion- related acute lung injury in mice. JExp Med. 2011;208(12):2525-2544.

9. Hilt ZT, et al. Platelet-derived $\beta 2 \mathrm{M}$ regulates monocyte inflammatory responses. JCI Insight. 2019;4(5):122943.

10. Koller BH, Marrack P, Kappler JW, Smithies O. Normal development of mice deficient in beta 2M, MHC class I proteins, and $\mathrm{CD} 8^{+} \mathrm{T}$ cells. 1990. J Immunol. 2010;184(9):4592-4595.

11. Zijlstra M, Bix M, Simister NE, Loring JM, Raulet $\mathrm{DH}$, Jaenisch R. Beta 2-microglobulin deficient mice lack CD4-8 ${ }^{+}$cytolytic T cells. Nature. 1990;344(6268):742-746. 
12. Looney MR, Su X, Van Ziffle JA, Lowell CA, Matthay MA. Neutrophils and their Fc gamma receptors are essential in a mouse model of transfusion-related acute lung injury. JClin Invest. 2006;116(6):1615-1623.

13. Ozato K, Mayer NM, Sachs DH. Monoclonal antibodies to mouse major histocompatibility complex antigens. Transplantation. 1982;34(3):113-120.

14. Kapur R, et al. T regulatory cells and dendritic cells protect against transfusion-related acute lung injury via IL-10. Blood. 2017;129(18):2557-2569.

15. Looney MR, Nguyen JX, Hu Y, Van Ziffle JA, Lowell CA, Matthay MA. Platelet depletion and aspirin treatment protect mice in a two-event model of transfusion-related acute lung injury. JClin Invest. 2009;119(11):3450-3461.

16. Alva JA, et al. VE-Cadherin-Cre-recombinase transgenic mouse: a tool for lineage analysis and gene deletion in endothelial cells. Dev Dyn. 2006;235(3):759-767.

17. Tiedt R, Schomber T, Hao-Shen H, Skoda RC. Pf4-Cre transgenic mice allow the generation of lineage-restricted gene knockouts for studying megakaryocyte and platelet function in vivo. Blood.2007;109(4):1503-1506.

18. Clausen BE, Burkhardt C, Reith W, Renkawitz $\mathrm{R}$, Förster I. Conditional gene targeting in macrophages and granulocytes using LysMcre mice. Transgenic Res. 1999;8(4):265-277.

19. Abram CL, Roberge GL, Hu Y, Lowell CA. Comparative analysis of the efficiency and specificity of myeloid-Cre deleting strains using ROSA-EYFP reporter mice. J Immunol Methods. 2014;408:89-100.

20. Yona $S$, et al. Fate mapping reveals origins and dynamics of monocytes and tissue macrophages under homeostasis. Immunity. 2013;38(1):79-91.

21. Ortiz-Muñoz G, Mallavia B, Bins A, Headley M, Krummel MF, Looney MR. Aspirin-triggered 15-epi-lipoxin A4 regulates neutrophil-platelet aggregation and attenuates acute lung injury in mice. Blood. 2014;124(17):2625-2634.

22. Murata K, Baldwin WM. Mechanisms of complement activation, $\mathrm{C} 4 \mathrm{~d}$ deposition, and their contribution to the pathogenesis of antibodymediated rejection. Transplant Rev (Orlando). 2009;23(3):139-150.

23. Toy P, et al. Transfusion-related acute lung injury: incidence and risk factors. Blood. 2012;119(7):1757-1767.

24. Lobo LJ, Aris RM, Schmitz J, Neuringer IP. Donor-specific antibodies are associated with antibody-mediated rejection, acute cellular rejection, bronchiolitis obliterans syndrome, and cystic fibrosis after lung transplantation. J Heart Lung Transplant. 2013;32(1):70-77.

25. Safavi S, Robinson DR, Soresi S, Carby M, Smith JD. De novo donor HLA-specific antibodies predict development of bronchiolitis obliterans syndrome after lung transplantation. J Heart Lung Transplant. 2014;33(12):1273-1281.

26. Morrell MR, et al. De novo donor-specific HLA antibodies are associated with early and highgrade bronchiolitis obliterans syndrome and death after lung transplantation.J Heart Lung Transplant. 2014;33(12):1288-1294.

27. Roux A, et al. Antibody-mediated rejection in lung transplantation: clinical outcomes and donor-specific antibody characteristics. Am J Transplant. 2016;16(4):1216-1228.

28. Tikkanen JM, et al. De novo DQ donor-specific antibodies are associated with chronic lung allograft dysfunction after lung transplantation. Am J Respir Crit Care Med. 2016;194(5):596-606.

29. Hachem RR, et al. Human leukocyte antigens antibodies after lung transplantation: Primary results of the HALT study. Am J Transplant. 2018;18(9):2285-2294.

30. Lefrançais E, Mallavia B, Zhuo H, Calfee CS, Looney MR. Maladaptive role of neutrophil extracellular traps in pathogen-induced lung injury. JCI Insight. 2018;3(3):98178.

31. Silliman CC, et al. Donor antibodies to HNA-3a implicated in TRALI reactions prime neutrophils and cause PMN-mediated damage to human pulmonary microvascular endothelial cells in a two-event in vitro model. Blood. 2007;109(4):1752-1755.

32. Abrahimi P, et al. Blocking MHC class II on human endothelium mitigates acute rejection. JCI Insight. 2016;1(1):e85293.

33. Brugière $\mathrm{O}$, et al. Role of $\mathrm{C} 1 \mathrm{q}-$ binding anti-HLA antibodies as a predictor of lung allograft outcome. Eur Respir J. 2018;52(2):1701898.

34. Bouquegneau A, et al. Complement-activating donor-specific anti-HLA antibodies and solid organ transplant survival: A systematic review and meta-analysis. PLoS Med. 2018;15(5):e1002572.

35. Torres-Ruiz J, et al. A potential role of neutrophil extracellular traps (NETs) in kidney acute antibody mediated rejection. Transpl Immunol. 2020;60:101286.

36. Caudrillier A, et al. Platelets induce neutrophil extracellular traps in transfusion-related acute lung injury. J Clin Invest. 2012;122(7):2661-2671.

37. Thomas GM, et al. Extracellular DNA traps are associated with the pathogenesis of TRALI in humans and mice. Blood. 2012;119(26):6335-6343.

38. Yuen J, et al. NETosing neutrophils activate complement both on their own NETs and bacteria via alternative and non-alternative pathways. Front Immunol. 2016;7:137.

39. Palmer LJ, Damgaard C, Holmstrup P, Nielsen $\mathrm{CH}$. Influence of complement on neutrophil extracellular trap release induced by bacteria. JPeriodont Res. 2016;51(1):70-76.

40. Sollberger G, et al. Gasdermin D plays a vital role in the generation of neutrophil extracellular traps. Sci Immunol. 2018;3(26): eaar6689.

41. Singh NR, Johnson A, Peters AM, Babar J, Chilvers ER, Summers C. Acute lung injury results from failure of neutrophil de-priming: a new hypothesis. Eur JClin Invest. 2012;42(12):1342-1349.

42. Carlin LM, et al. Nr4a1-dependent Ly6C(low) monocytes monitor endothelial cells and orchestrate their disposal. Cell. 2013;153(2):362-375.

43. Jongerius I, et al. The role of complement in transfusion-related acute lung injury. Transfus Med Rev. 2019;33(4):236-242.

44. McKenzie CG, Kim M, Singh TK, Milev Y, Freedman J, Semple JW. Peripheral blood monocyte-derived chemokine blockade prevents murine transfusion-related acute lung injury (TRALI). Blood. 2014;123(22):3496-3503.

45. Sreeramkumar V, et al. Neutrophils scan for acti- vated platelets to initiate inflammation. Science. 2014;346(6214):1234-1238.

46. Hori M, Kitakaze M. Adenosine, the heart, and coronary circulation. Hypertension. 1991;18(5):565-574

47. McVey MJ, et al. Acid sphingomyelinase mediates murine acute lung injury following transfusion of aged platelets. Am J Physiol Lung Cell Mol Physiol. 2017;312(5):L625-L637.

48. Hechler B, et al. Platelets are dispensable for antibody-mediated transfusion-related acute lung injury in the mouse. J Thromb Haemost. 2016;14(6):1255-1267.

49. Beutier $\mathrm{H}$, et al. Platelets expressing IgG receptor Fc $\gamma$ RIIA/CD32A determine the severity of experimental anaphylaxis. Sci Immunol. 2018;3(22):eaan5997.

50. Mnjoyan Z, Li J, Afshar-Kharghan V. Factor H binds to platelet integrin alphaIIbbeta3. Platelets. 2008;19(7):512-519.

51. Del Conde I, Crúz MA, Zhang H, López JA, Afshar-Kharghan V. Platelet activation leads to activation and propagation of the complement system. J Exp Med. 2005;201(6):871-879.

52. Sauter RJ, et al. Functional relevance of the anaphylatoxin receptor $\mathrm{C} 3 \mathrm{aR}$ for platelet function and arterial thrombus formation marks an intersection point between innate immunity and thrombosis. Circulation. 2018;138(16):1720-1735.

53. Yuan Y, et al. Neutrophil macroaggregates promote widespread pulmonary thrombosis after gut ischemia. Sci Transl Med. 2017;9(409):eaam5861.

54. Cleary SJ, et al. LPS-induced lung platelet recruitment occurs independently from neutrophils, PSGL-1, and P-selectin. Am J Respir Cell Mol Biol. 2019;61(2):232-243.

55 . Morrell CN, et al. In vivo platelet-endothelial cell interactions in response to major histocompatibility complex alloantibody. Circ Res. 2008;102(7):777-785.

56. Schnütgen F, Doerflinger N, Calléja C, Wendling O, Chambon P, Ghyselinck NB. A directional strategy for monitoring Cre-mediated recombination at the cellular level in the mouse. Nat Biotechnol. 2003;21(5):562-565.

57. Headley MB, et al. Visualization of immediate immune responses to pioneer metastatic cells in the lung. Nature. 2016;531(7595):513-517.

58. Lefrançais $\mathrm{E}$, et al. The lung is a site of platelet biogenesis and a reservoir for haematopoietic progenitors. Nature. 2017;544(7648):105-109.

59. Mallavia B, et al. Mitochondrial DNA stimulates TLR9-dependent neutrophil extracellular trap formation in primary graft dysfunction. Am J Respir Cell Mol Biol. 2020;62(3):364-372.

60. Meyer KC, et al. An international ISHLT/ATS/ ERS clinical practice guideline: diagnosis and management of bronchiolitis obliterans syndrome. Eur Respir J. 2014;44(6):1479-1503.

61. Calabrese DR, et al. NKG2C natural killer cells in bronchoalveolar lavage are associated with cytomegalovirus viremia and poor outcomes in lung allograft recipients. Transplantation. 2019;103(3):493-501.

62. Calabrese DR, et al. Dectin-1 genetic deficiency predicts chronic lung allograft dysfunction and death. JCI Insight. 2019;4(22):133083. 